\title{
Mixed-Integer Cuts from Cyclic Groups
}

\author{
Matteo Fischetti and Cristiano Saturni \\ DEI, Department of Information Engineering, \\ University of Padova, \\ via Gradenigo 6/A, \\ I35126 Padova, Italy \\ e-mail: \{matteo.fischetti, cristiano.saturni\}@unipd.it
}

September 10, 2005

\begin{abstract}
We analyze a separation procedure for Mixed-Integer Programs related to the work of Gomory and Johnson on interpolated subadditive functions. This approach has its roots in the Gomory-Johnson characterization on the master cyclic group polyhedron. To our knowledge, the practical benefit that can be obtained by embedding interpolated subadditive cuts in a cutting plane algorithm was not investigated computationally by previous authors. In this paper we compute, for the first time, the lower bound value obtained when adding (implicitly) all the interpolated subadditive cuts that can be derived from the individual rows of an optimal LP tableau, thus approximating the optimization over the Gomory's corner polyhedron. The computed bound is compared with that obtained when only Gomory mixed-integer cuts are used, on a very large test-bed of MIP instances.
\end{abstract}

Key words: Mixed-Integer Programming, Subadditive cuts, Gomory cuts, Gyclic Group and Corner polyhedra.

\section{Introduction}

In this paper we study the Integer Linear Program (ILP)

$$
\min \left\{c^{T} x: A x=b, x \geq 0 \text { integer }\right\}
$$

where $A$ is a rational $m \times n$ matrix, $b$ is a rational $m$-dimensional vector, and $c \in \mathcal{R}^{n}$ is a cost vector, and we address the two associated polyhedra:

$$
\begin{aligned}
& P:=\left\{x \in \mathcal{R}_{+}^{n}: A x=b\right\} \\
& P_{I}:=\operatorname{conv}\left\{x \in \mathcal{Z}_{+}^{n}: A x=b\right\}=\operatorname{conv}\left(P \cap \mathcal{Z}^{n}\right) .
\end{aligned}
$$

The mixed-integer case where some variables are not restricted to be integer, will be addressed in Section 3 . 
We propose an exact separation procedure for the class of so-called interpolated (or template) subadditive cuts, based on the characterization of Gomory and Johnson $[12,13,14]$ of the master cyclic group polyhedron defined as

$$
T(k, r)=\operatorname{conv}\left\{t \in \mathcal{Z}_{+}^{k-1}: \sum_{i=1}^{k-1}(i / k) \cdot t_{i} \equiv r / k(\bmod 1)\right\}
$$

where $k \geq 2$ (group order) and $r \in\{1, \cdots, k-1\}$ are given integers. The space $\mathcal{R}^{k-1}$ of the $t$ variables is called the $T$-space in [15]. It is known that the mapping the original $x$-variable space into the $T$-space allows one to use polyhedral information on $T(k, r)$ to derive valid inequalities for $P_{I}$. To our knowledge, however, the practical benefit that can be obtained by embedding the whole family of cyclic-group cuts in a cutting plane algorithm was not investigated computationally by previous authors. As a matter of fact, a number of recent papers $[15,16,6,7,8,3]$ deals only implicitly with cyclic-group separation, as they address the so-called Gomory's shooting experiment. Roughly speaking, in this experiment the point $t^{*} \in \mathcal{R}^{k-1}$ to be separated is generated at random (hence corresponding to a random "shooting direction" in the $T$-space), and statistics on the frequency of the most-violated facets of $T(k, r)$ are collected. A very recent paper presenting some computational results is the one by Koppe, Louveaux, Weismantel and Wolsey [20], where a compact formulation of the cyclic-group separation problem is embedded into the original ILP model - this however produces a huge extended formulation with limited practical applications. Also related to our work are the papers by Cornuejols, Li and Vandenbussche [5], where a subfamily of cyclic-group cuts (called $k$-cuts) is investigated both theoretically and computationally, and by Letchford and Lodi [19], where a different subfamily is addressed.

This paper is organized as follows. In Section 2 we present the theory of Gomory and Johnson [12, 13, 14] on interpolated subadditive functions (called template functions in [7]) and their role in generating valid inequalities for $P_{I}$. We also introduce an exact separation procedure for interpolated subadditive cuts based on an LP model taken from the Gomory-Johnson characterization of the master cyclic group polyhedron. This separation procedure allows us to exploit effectively the whole family of interpolated subadditive cuts to improve the LP relaxation quality. In Section 3 we consider the mixed-integer case, where model (1) becomes a MIP involving continuous variables, whereas in Section 4 we address some implementation issues related to the presence of bounded variables. In Section 5, the quality of the generated cuts is analyzed computationally. In particular we compute, for the first time, the lower bound value obtained when adding (implicitly) all the interpolated subadditive cuts that can be derived from the individ- 
ual rows of an optimal LP tableau. This leads to an approximation ${ }^{1}$ of the optimization over of the Gomory's corner polyhedron [14], thus giving a partial answer to the question posed in [16] (and also addressed in [20]) on the quality of this relaxation. The bound we compute is compared with that obtained when only Gomory mixed-integer cuts are used, on a very large test-bed of MIP instances [21] library. The outcome is that Gomory mixed-integer cuts play a very special role among subadditive cuts, in that they typically produce, alone, a lower bound increase which is comparable to that obtained when the whole family of cuts is considered. This result confirms the theoretical findings of Dash and Gunluk [7], who showed that interpolated subadditive cuts are dominated by Gomory mixed-integer cuts in a probabilistic sense, as well as the computational experience of Cornuejols, $\mathrm{Li}$ and Vandenbussche [5] on the subfamily of $k$-cuts. Some interesting directions of work are finally addressed in Section 6 .

An earlier version of the present paper was presented at the IPCO XI meeting held in Berlin, June 2005.

\section{Cuts from Subadditive Functions}

The fractional part $\phi(a)$ of a real value $a$ is defined as

$$
\phi(a):=a-\lfloor a\rfloor,
$$

where $\lfloor a\rfloor$ denotes the largest integer not greater than $a$. Given a positive integer $k$ and two real values $a, b \in \mathcal{R}$, we write $a \equiv b(\bmod k)$ if $a-b$ is an integer multiple of $k$. In this paper we are interested in deriving valid inequalities for $P_{I}$ that are not implied by the system $A x=b, x \geq 0$. To this end, given any equation

$$
\alpha^{T} x=\beta
$$

valid for $P_{I}$, where $(\alpha, \beta) \in \mathcal{Q}^{n+1}$ and $\phi(\beta)>0$, we consider the group polyhedron

$$
G(\alpha, \beta):=\operatorname{conv}\left\{x \in \mathcal{Z}_{+}^{n}: \sum_{j=1}^{n} \alpha_{j} x_{j} \equiv \beta(\bmod 1)\right\} \supseteq P_{I} .
$$

For example, the equation $\alpha^{T} x=\beta$ can be obtained by setting $(\alpha, \beta)^{T}:=$ $u^{T}(A, b)$ for any $u \in \mathcal{Q}^{m}$ such that $\phi\left(u^{T} b\right)>0$. This is the case, e.g., when the equation is read from the tableau associated with a fractional optimal solution of the LP relaxation of (1).

In particular, we address the following separation problem:

\footnotetext{
${ }^{1}$ Besides getting rid of the effects of interpolation, optimizing exactly over the corner polyhedron would require to take into account all the tableau rows simultaneously.
} 
Definition (g-SEP) Given any point $x^{*} \geq 0$ and the equation $\alpha^{T} x=\beta$ with rational coefficients and such that $\phi(\beta)>0$, find (if any) a valid inequality for $G(\alpha, \beta)$ that is violated by $x^{*}$.

Notice that, as far as $G(\alpha, \beta)$ is concerned, one can replace each entry in $(\alpha, \beta)$ by its fractional part, hence one can assume without loss of generality $0 \leq \alpha_{j}<1$ for all $j$, and $0<\beta<1$.

A function $g: \mathcal{R} \rightarrow \mathcal{R}$ is called subadditive if $g(a+b) \leq g(a)+g(b)$ for any $a, b \in \mathcal{R}$. We call a subadditive function $g(\cdot)$ periodic in $[0,1)$ if $g(a+1)=g(a)$ for all $a \in \mathcal{R}$. As in this paper we are only interested in subadditive functions $g(\cdot)$ that are periodic in $[0,1)$ and such that $g(0)=0$, in the sequel we will name this kind of functions just subadditive.

Given a valid equation $\alpha^{T} x=\beta$ for $P_{I}$, it is easy to show that the inequality

$$
\sum_{j=1}^{n} g\left(\alpha_{j}\right) x_{j} \geq g(\beta)
$$

is valid for $G(\alpha, \beta)$ (and hence for $P_{I}$ ) whenever $g(\cdot)$ is subadditive. For example, taking $g(\cdot)=\phi(\cdot)$ one obtains the well-know Gomory fractional cut [10]

$$
\sum_{j=1}^{n} \phi\left(\alpha_{j}\right) x_{j} \geq \phi(\beta)
$$

whereas taking the subadditive GMI function $\gamma^{\beta}(\cdot)$ defined as

$$
\gamma^{\beta}(a)=\left\{\begin{array}{ll}
\phi(a) & \text { if } \phi(a) \leq \phi(\beta) \\
\phi(\beta) \frac{1-\phi(a)}{1-\phi(\beta)} & \text { otherwise }
\end{array} \quad \text { for all } a \in \mathcal{R}\right.
$$

one obtains the stronger Gomory Mixed-Integer (GMI) cut [11]

$$
\sum_{j=1}^{n} \min \left\{\phi\left(\alpha_{j}\right), \phi(\beta) \frac{1-\phi\left(\alpha_{j}\right)}{1-\phi(\beta)}\right\} x_{j} \geq \phi(\beta) .
$$

see Figure 1 for an illustration.

A basic result, due to Gomory and Johnson [13, 14], is that all the nontrivial facets ${ }^{2}$ of $G(\alpha, \beta)$ are defined by inequalities of this type. ${ }^{3}$ As a consequence, our separation problem $(g-S E P)$ can be rephrased as the problem of defining a suitable subadditive function $g(\cdot)$ that produces a cut violated by the given point $x^{*}$, i.e., such that $\sum_{j=1}^{n} g\left(\alpha_{j}\right) x_{j}^{*}<g(\beta)$.

\footnotetext{
${ }^{2} \mathrm{~A}$ facet if called nontrivial if it is not defined by a nonnegativity constraint

${ }^{3}$ In addition, Gomory and Johnson have shown that non-dominated inequalities only arise when the complementarity condition $g(a)+g(b)=g(a+b)(=g(\beta))$ holds whenever $a+b \equiv \beta(\bmod 1)$.
} 
(a)

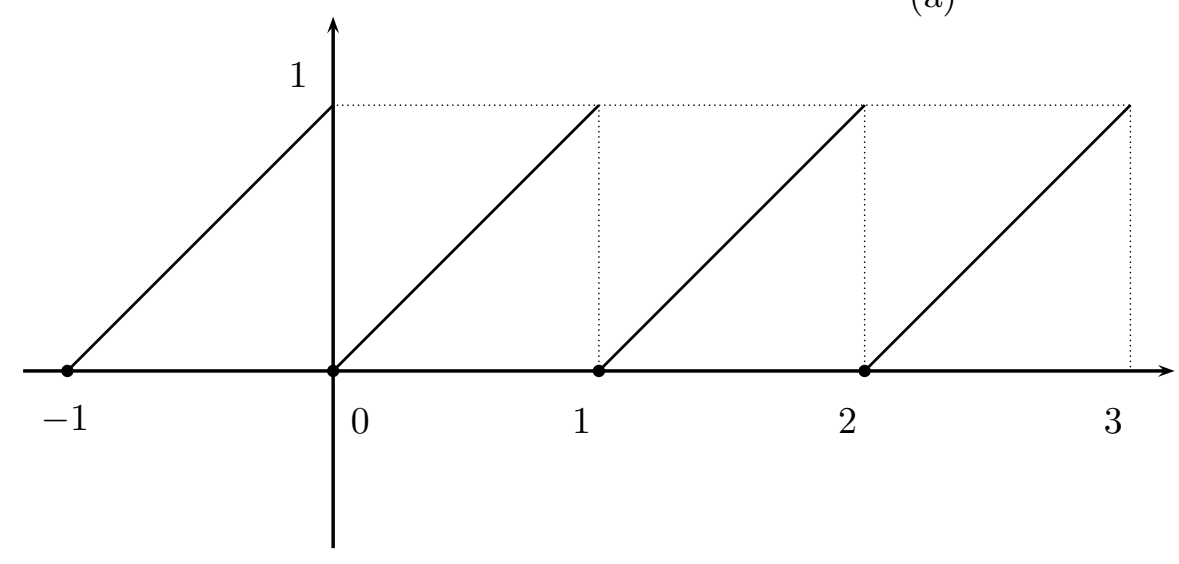

(b)

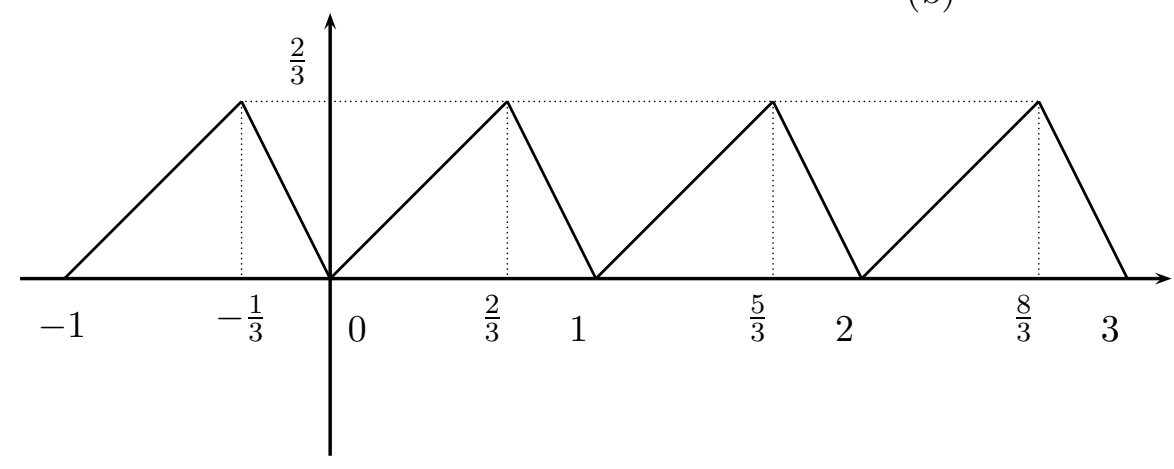

Figure 1: Two subadditive functions: the fractional part $\phi(\cdot)$ (top) and the GMI function $\gamma^{2 / 3}(\cdot)$ (bottom) 
Now let $k \geq 2$ be the smallest integer such that $k(\alpha, \beta)$ is integer, whose existence follows from the assumption that $(\alpha, \beta)$ is rational. This value of $k$ will be called ideal with respect to equation (5). Of course, the subadditivity (plus periodicity) of $g(\cdot)$ implies that the same property holds over the discrete set $\{0,1 / k, 2 / k, \cdots,(k-1) / k\}$. In other words, a necessary condition for subadditivity is that the "sampled" values $g_{i}:=g(i / k), i=0, \cdots, k-1$ satisfy the following set of linear constraints, called $g$-system in the sequel:

$$
\begin{aligned}
& g_{h} \leq g_{i}+g_{j}, \quad 1 \leq i, j, h \leq k-1 \text { and } i+j \equiv h(\bmod k) \\
& g_{0}=0 \\
& 0 \leq g_{i} \leq 1, \quad i=1, \cdots, k-1
\end{aligned}
$$

where bounds (12) will play a normalization role in the sequel.

Any solution $\left(g_{0}, \cdots, g_{k-1}\right)$ of the $g$-system above can be completed so as to define a subadditive function $g: \mathcal{R} \rightarrow \mathcal{R}$ through a simple interpolation procedure due to Gomory and Johnson [13]. This procedure simply takes a linear interpolation of the values $g_{0}, \cdots, g_{k-1}$ over $[0,1)$, and then extends the resulting piecewise-linear function to $\mathcal{R}$, in the obvious periodic way. More formally, for any $a \in \mathcal{R}$ the interpolated value $g(a)$ is defined as $g(a)=(1-\theta) g_{i}+\theta g_{i+1}$, where $\theta \in[0,1)$ and $i \in\{0, \cdots, k-1\}$ are such that $\phi(a)=(1-\theta) i / k+\theta(i+1) / k$, and $g_{k}:=g_{0}$ because of periodicity.

A key observation at this point is that, being $k$ ideal, the actual value of $g(\cdot)$ outside the sample points $i / k$ is immaterial, since $g(\cdot)$ only needs to be evaluated on these sample points when computing the coefficients in (7). Therefore, the interpolation procedure does not actually restrict the space of the possible subadditive functions - as it would be the case for a different choice of $k$. As a consequence, we can exactly rephrase $g$-SEP as the following LP:

$$
g-S E P_{k}: \quad \min \left\{\sum_{i=1}^{k-1} t_{i}^{*} g_{i}:(10)-(12)\right\},
$$

where $r:=k \phi(\beta), t_{i}^{*}:=\sum_{j}\left\{x_{j}^{*}: \phi\left(\alpha_{j}\right)=i / k\right\}$ for $i \in\{1, \cdots, k-1\} \backslash\{r\}$, and $t_{r}^{*}:=\sum_{j}\left\{x_{j}^{*}: \phi\left(\alpha_{j}\right)=r / k\right\}-1$ so as to take into account the role of the right hand side $g(\beta)=g_{r}$ in (7). With these definitions, the objective function $\sum_{i=1}^{k-1} t_{i}^{*} g_{i}$ is precisely the opposite of the violation of a cut of the form (7), hence a violated such cut exists if and only if the optimal value of $g-S E P_{k}$ is strictly negative.

Unfortunately, the ideal $k$ is very often too large to be used in practice, so one has to choose a smaller value in order to produce a manageable $g$-system. In this case, the interpolation procedure does restrict (often considerably) the range of subadditive functions that can be captured by $g-S E P_{k}$. Moreover, for a non-ideal $k$ the definition of the weights $t_{i}^{*}$ becomes slightly more involved, due to the need of taking interpolation into account. More specifically, for any integer $k \geq 2$ (not necessarily ideal) the weights $t_{i}^{*}$ in (13) are 


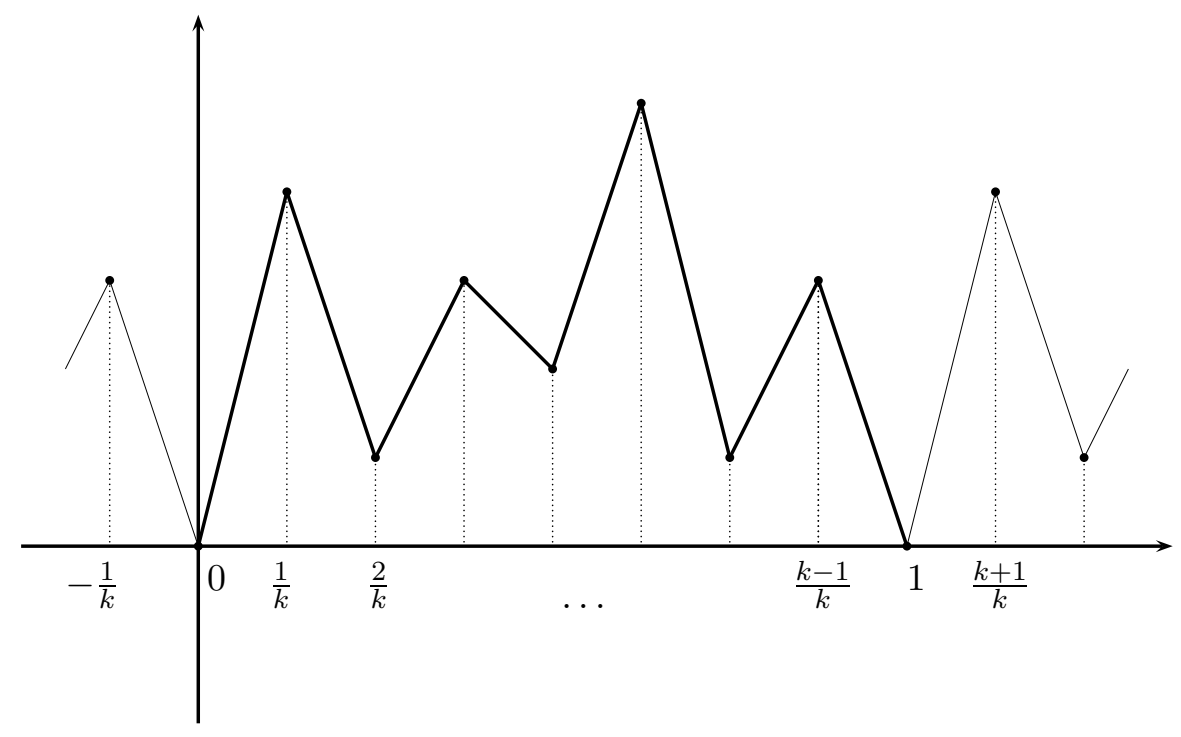

Figure 2: The Gomory-Johnson interpolation procedure: the sample values $g(0), g(1 / k) \cdots, g((k-1) / k)$ are connected by straight lines so to get the subadditive function $g(\cdot)$ over $[0,1)$, which is then extended periodically over $\mathcal{R}$.

defined through the algorithm outlined in Figure 3, that works as follows. At step 1, we define two fictitious values $\alpha_{0}$ and $x_{0}^{*}$ so as to re-write the (opposite of the) cut violation $\sum_{j=1}^{n} g\left(\alpha_{j}\right) x_{j}^{*}-g(\beta)$ in the more convenient form $\sum_{j=0}^{n} g\left(\alpha_{j}\right) x_{j}^{*}$. At step 2, all weights $t_{i}^{*}$ are initially set to zero. At step 3 , for each $j=0, \cdots, n$ we locate the interval $[i / k,(i+1) / k)$ that contains $\phi\left(\alpha_{j}\right)$, where $i+1$ is replaced by 0 in case $i+1=k$ so as to take periodicity into account. At step 4 we define a "displacement" parameter $\theta \in[0,1)$ giving the exact position of $\phi\left(\alpha_{j}\right)$ within this interval; by definition, we have $\theta=0$ if $\phi\left(\alpha_{j}\right)=i / k$, whereas $\theta$ approaches its limit 1 as $\phi\left(\alpha_{j}\right)$ approaches $(i+1) / k$. We then split, at step 5 , the contribution $g\left(\alpha_{j}\right) x_{j}^{*}$ between $t_{i}^{*}$ and $t_{i+1}^{*}$, in a way proportional to $\theta$. Note that the procedure also works in case of ideal $k$, where we always have $\theta=0$ at step 4 .

We finally observe that, for the interpolated function $g(\cdot)$, we sometimes have $g(a)>g(\beta)$ as, e.g., in the case illustrated in Figure 4. In this figure we compare a GMI function and the corresponding interpolated version for $k=3$ (top), and scale them so as to get the same right-hand-side value (bottom) so as to make clear the fact that the interpolated function produces a dominated inequality. Therefore, an interpolated subadditive cut 
1. define the fictitious values $\alpha_{0}:=\beta$ and $x_{0}^{*}:=-1$;

2. initialize $t_{0}^{*}:=t_{1}^{*}:=\cdots:=t_{k-1}^{*}:=0$;

2. for $j=0,1, \cdots, n$ such that $x_{j}^{*}>0$ and $\phi\left(\alpha_{j}\right)>0$ do

3. let $i:=\left\lfloor k \phi\left(\alpha_{j}\right)\right\rfloor$ and $h=i+1 \bmod k$;

4. let $\theta:=k \phi\left(\alpha_{j}\right)-i$;

5. update $t_{i}^{*}:=t_{i}^{*}+(1-\theta) x_{j}^{*}$ and $t_{h}^{*}:=t_{h}^{*}+\theta x_{j}^{*}$

6. enddo

Figure 3: Defining the weights $t_{i}^{*}$ in $g-S E P_{k}$ for any given $k$ and $x^{*}$

$\sum_{j=1}^{n} g\left(\alpha_{j}\right) x_{j} \geq g(\beta)$ can easily be improved to its clipped form:

$$
\sum_{j=1}^{n} \min \left\{g\left(\alpha_{j}\right), g(\beta)\right\} x_{j} \geq g(\beta)
$$

whose validity follows trivially from the integrality of $x$.

\section{Dealing with Continuous Variables}

We next address the case where some variables $x_{j}$ with $j \in \mathcal{C}$ (say) are not restricted to be integer valued. In this case, Gomory and Johnson [13, 14] showed that, for any subadditive function $g(\cdot)$, it is enough to modify cut (7) into

$$
\sum_{j \in \mathcal{I}}^{n} g\left(\alpha_{j}\right) x_{j}+\sum_{j \in \mathcal{C}: \alpha_{j}>0} \text { slope }_{+} \alpha_{j} x_{j}+\sum_{j \in \mathcal{C}: \alpha_{j}<0} \text { slope }_{-} \alpha_{j} x_{j} \geq g(\beta),
$$

where $\mathcal{I}:=\{1, \cdots, n\} \backslash \mathcal{C}$ is the index set of the integer-valued variables,

$$
\text { slope }_{+}:=\lim _{\delta \rightarrow 0^{+}} g(\delta) / \delta
$$

is the slope of $g(\cdot)$ in $0^{+}$, and

$$
\text { slope }_{-}:=\lim _{\delta \rightarrow 0^{-}} g(\delta) / \delta=-\lim _{\delta \rightarrow 0^{+}} g(1-\delta) / \delta
$$

is the slope of $g(\cdot)$ in $0^{-}$(or, equivalently, in $1^{-}$). Notice that, by definition, slope $_{+}>0$ and slope $_{-}<0$, hence all coefficients in (15) are nonnegative.

The above result has an intuitive explanation based on the following simple scaling argument. Let $j \in \mathcal{C}$ be the index of any continuous variable. We introduce a scaled copy $\tilde{x}_{j}=M x_{j}$ of $x_{j}$, where $M>0$ is a suitable scaling factor, and impose that $\tilde{x}_{j}$ can only assume integer values. This is of course correct only if $M$ is chosen so as not to cut any feasible point of the 

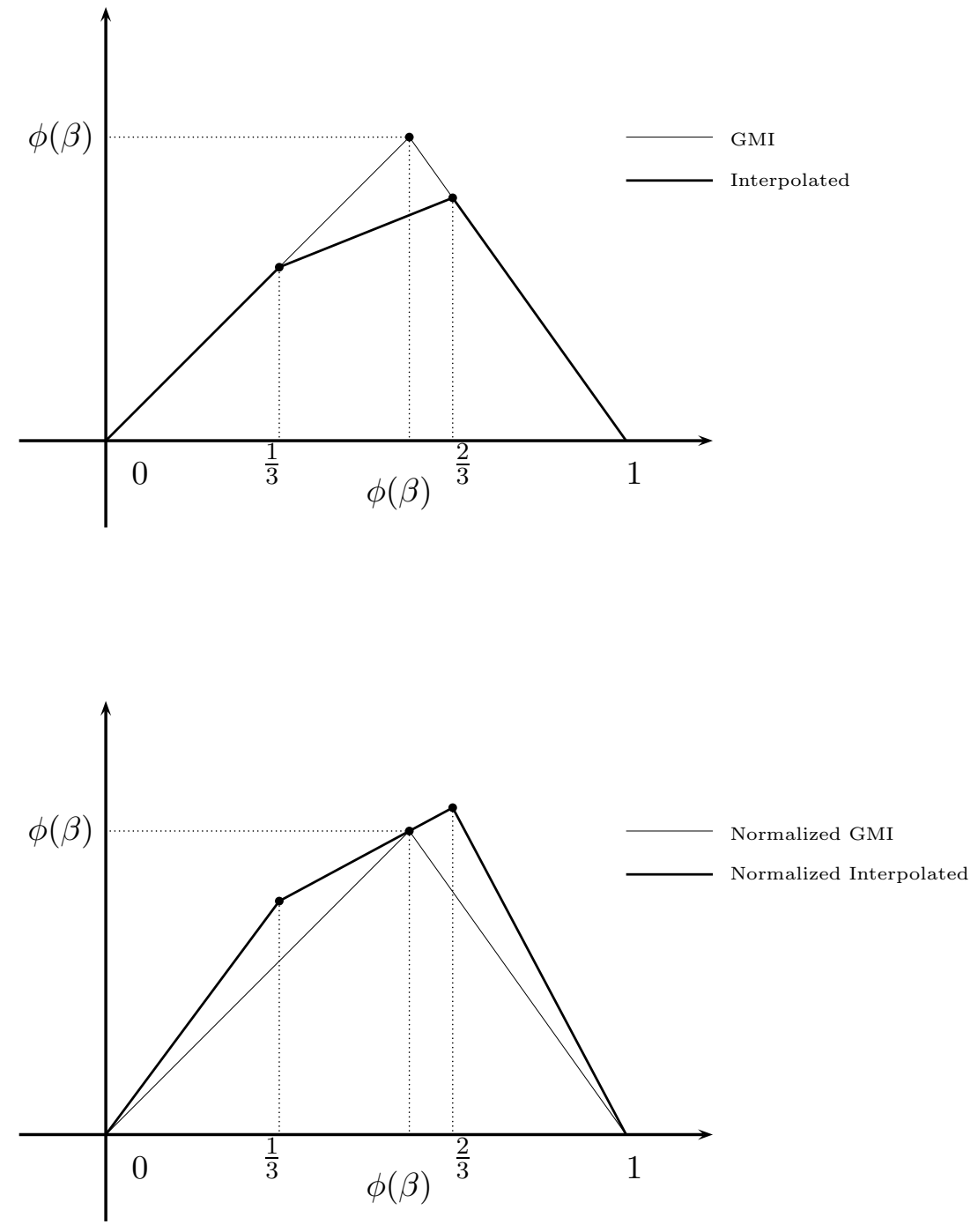

Figure 4: GMI and interpolated GMI functions 
original MIP set, which is always possible due the rational data assumption. Notice that $M$ can be assumed to be arbitrarily large, since multiplying a valid $M$ by a positive integer yields another valid $M$. Now, replacing $x_{j}$ by $\tilde{x}_{j} / M$ changes the $j$-th coefficient in equation $\alpha^{T} x=\beta$ from $\alpha_{j}$ to $\tilde{\alpha}_{j}:=\alpha_{j} / M$, while increasing the $j$-th component of $x^{*}$ from $x_{j}^{*}$ to $\tilde{x}_{j}^{*}:=$ $M x_{j}^{*}$. Being $\tilde{x}_{j}$ constrained to be integer, we can compute its coefficient in $(7)$ as $g\left(\tilde{\alpha}_{j}\right)=g\left(\alpha_{j} / M\right)$. If $\alpha_{j}>0$, for $M \rightarrow+\infty$ we have that $\alpha_{j} / M$ tends to $0^{+}$, hence for sufficiently large $M$ we have $g\left(\tilde{\alpha}_{j}\right)=g\left(\alpha_{j} / M\right)=$ slope $_{+} \alpha_{j} / M$. Analogously, if $\alpha_{j}<0$ we have $\alpha_{j} / M \rightarrow 0^{-}$when $M \rightarrow+\infty$, hence for sufficiently large $M$ we have $g\left(\tilde{\alpha}_{j}\right)=g\left(\alpha_{j} / M\right)=$ slope $_{-} \alpha_{j} / M$. This shows that the coefficient of the scaled variable $\tilde{x}_{j}$ in the subadditive

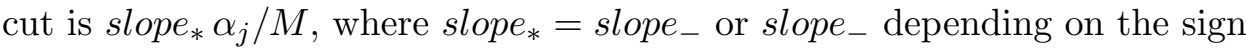
of $\alpha_{j}$. The back substitution $x_{j}=\tilde{x}_{j} / M$ then yields the coefficient slope $_{*} \alpha_{j}$ for the original (continuous) variable $x_{j}$, as in (15).

As a consequence of the above scaling argument, we can deal with continuous variables without any modification of our separation procedure, that can used as a black box. To this end, it is enough to implement a pre-processing scaling phase for the continuous variables, and then a postprocessing phase where the separated cut returned by the black box is expressed in terms of the original (non-scaled) variables. In case of interpolated subadditive functions, a suitable scaling factor for each continuous variable $x_{j}$ is $M_{j}:=k\left|\alpha_{j}\right|$, that maps the original coefficient $\alpha_{j}$ into $\tilde{\alpha}_{j}=\alpha_{j} / M_{j}= \pm 1 / k$.

Alternatively, one can modify slightly the separation procedure of Figure 3 so as to take into account continuous variables in an explicit way. To this end, we observe that for interpolated subadditive functions with interpolation values $g_{i}:=g(i / k)$ for $i=0, \cdots, k-1$ and $g_{0}=0$, one has slope $_{+}=g_{1} /(1 / k)$ and slope $-=-g_{k-1} /(1 / k)$. Hence, in the definition of the weights $t_{i}^{*}$ used in the separation problem (13) the value $x_{j}^{*}$ of a continuous variable $x_{j}(j \in \mathcal{C})$ contributes to $t_{1}^{*}$ or $t_{k-1}^{*}$, depending on the sign of $\alpha_{j}$. To be more specific, for each $j \in \mathcal{C}, j \neq 0$, one has to skip steps 3-4 in Figure 3, and update $t_{1}^{*}:=t_{1}^{*}+k\left|\alpha_{j}\right| x_{j}^{*}$ in case $\alpha_{j}>0$, and $t_{k-1}^{*}:=t_{k-1}^{*}+k\left|\alpha_{j}\right| x_{j}^{*}$ otherwise.

The above considerations show that, in presence of several continuous variables with nonzero $\alpha_{j} x_{j}^{*}$, values $g_{1}$ and $g_{k-1}$ play a crucial role in the separation, the lower these values the better. Hence GMI cuts qualify as the strongest subadditive cuts when continuous variables are present, since they have the property of being associated with a subadditive function $\gamma^{\beta}(\cdot)$ where slope + and slope $e_{-}$are as small as possible; see [14].

Finally, we observe that the clipping of coefficient $g\left(\alpha_{j}\right)$ to $\min \left\{g\left(\alpha_{j}\right), g(\beta)\right\}$ in (14) is guaranteed to be valid only for integer-constrained variables $x_{j}$, so it cannot be applied for $j \in \mathcal{C}$. 


\section{Dealing with bounded variables}

It is often the case that the original MIP model involves variable bounds of the form $x_{j} \leq U B_{j}$ for some variables $x_{j}$. Commercial LP solvers typically deal with variable upper bounds in an implicit way, hence the tableau rows are not exactly the same as in the textbook theory. (E.g., due to the presence of nonbasic variables at their upper bound, a basic fractional variable is sometimes associated with a tableau row whose right-hand-side value is integer.) We next outline a possible way to handle bounded variables.

Given any equation $\alpha^{T} x=\beta$ we address the possibility of complementing some bounded variables $x_{j}$ (i.e., replacing $x_{j}$ by $U B_{j}-x_{j}$ ) before actually invoking the separator. Of course, an optimal choice of the variables to be complemented can improve the chances of finding a violated cut, but this choice does not appear easy.

To illustrate this point, let us consider a pure 0-1 problem where all variables are bounded by 1 , and let $\sum_{j=1}^{n} \alpha_{j} x_{j}=\beta$ by any "knaspack" equation valid for $P_{I}$. Without loss of generality one can assume $0 \leq \alpha_{j} \leq$ $\beta \leq 1$ for all $j$. A set $Q \subseteq\{1, \cdots, n\}$ with $\sum_{j \in Q} \alpha_{j}>\beta$ is called a cover. Given any minimal (with respect to set inclusion) cover $Q$, we complement the corresponding variables by replacing $x_{j}$ by $1-\bar{x}_{j}$, and obtain

$$
\sum_{j \in Q} \alpha_{j} \bar{x}_{j}+\sum_{j \notin Q}\left(-\alpha_{j}\right) x_{j}=\sum_{j \in Q} \alpha_{j}-\beta=: \theta>0
$$

where $\theta<1$ and $\alpha_{j}>0$ for all $j \in Q$ because of the minimality of the cover. Now take the subadditive function $g(a):=\lceil\phi(a)\rceil-\phi(a)$, and observe that $0<\alpha_{j}<1$ implies $g\left(\alpha_{j}\right)=1-\alpha_{j}$ and $g\left(-\alpha_{j}\right)=g\left(1-\alpha_{j}\right)=1-\phi\left(1-\alpha_{j}\right)=$ $1-\left(1-\alpha_{j}\right)=\alpha_{j}$. Applying $g(\cdot)$ to $(16)$ we then obtain the cut

$$
\sum_{j \in Q}\left(1-\alpha_{j}\right) \bar{x}_{j}+\sum_{j \notin Q} \alpha_{j} x_{j} \geq g(\theta)=1-\theta
$$

and adding together (16) and (17) we finally get the so-called cover inequality $[22]$

$$
\sum_{j \in Q} \bar{x}_{j}=\sum_{j \in Q}\left(1-x_{j}\right) \geq 1
$$

Note that one can have exponentially many different cover inequalities, each associated with a different subset $Q$ of complemented variables.

The above example suggests that there is no easy way to determine the best set of variables to be complemented, just as there is no easy way to locate the set $Q$ that produces a most-violated cover inequality when dealing with knapsack constraints. Hence some heuristics have to be applied. A natural choice (also used by other authors) is to complement only the variables that assume the LP status "nonbasic at its upper bound" in the corresponding optimal solution. This guarantees that the equations inserted 
in our pool have the familiar "textbook form" where all nonbasic variables $x_{j}$ have value $x_{j}^{*}=0$ with respect to the basic LP solution $x^{*}$ of the same tableau.

\section{Computational Results}

We next report a computational analysis aimed at comparing the quality of Gomory mixed-integer cuts with that of the interpolated sudadditive cuts, when embedded in a pure cutting plane method. We also report a comparison with an important class of (non-interpolated) subadditive cuts, namely, the $k$-cuts described in [5].

Our test-bed includes all MIPLIB 3.0 and 2003 instances taken form [21], except those with unknown optimal solution or having some variables with negative lower bound (we also excluded from our test-bed some very large instances, namely, all those having an LP file larger than than 1.7 MB). In addition, we addressed the hard ILP instances available at the Alper Atamtürk's home page [2], associated with multiple-knapsack problems involving both binary and general-integer (either bounded or unbounded) variables. Finally, our test-bed includes a set of random (both bounded and binary) single-knapsack problems generated as in [5], namely:

$$
\begin{aligned}
& \min \sum_{i=1}^{n} p_{i} x_{i} \\
& \text { s.t. } \sum_{i=1}^{n} w_{i} x_{i} \leq c \\
& 0 \leq x_{i} \leq b_{i} \text { and integer for all } i=1 \ldots n
\end{aligned}
$$

with $p_{i}$ and $w_{i}$ uniformly random integers in $[1,1000], b_{i}$ uniformly random integers in $[5,10]$ and $c=\left\lfloor 0.5 \sum_{i=1}^{n} w_{i} b_{i}\right\rfloor$. Binary knapsack problems were generated in the same way, by setting $b_{i}=1$ for all $i$.

For the problems involving " $\leq$ " or " $\geq$ " constraints we built an equivalent formulation in standard form, that includes slack variables in an explicit way. The bounds on the variables, instead, were dealt with in an implicit way, as outlined in Section 4.

All LP's were solved through the commercial software ILOG-Cplex 9.0 $[17,18]$. Computing times are expressed in CPU seconds and refer to a notebook with a 512MB RAM and a 1.6Mhz AMD Processor.

Our order of business was to approximate the optimization over the Gomory's corner polyhedron associated with the optimal solution of the LP relaxation of our MIP model (without any MIP preprocessing). To this end, after the solution of the first LP relaxation of our model, we stored in an equation pool all the tableau rows $\alpha^{T} x=\beta$ with fractional right-hand side $\beta$, along with the list of the variables that are at their upper bound in the optimal LP solution (these variables were always complemented before invoking our separation procedures, no matter their value in the current point 
$x^{*}$ to be separated). Neither the pool nor the list of complemented variables was updated during the run, i.e., we deliberately avoided generating subadditive cuts of rank greater than 1 (or, to be more precise, we avoid cuts derived from equations different from those associated with the single rows of the first LP tableau). The same applies to Gomory mixed-integer cuts, that were derived from the equations in the pool and added (at once) to the LP. At each round of separation, at most 200 cuts were generated. Each run was aborted at the root node, i.e., no branching was allowed.

The outcome of our experiments on the MIPLIB istances is shown in Tables 1-4 (mixed-integer problems) and 5-6 (pure-integer problems). In these tables, the first numerical value under the name of the problem gives the optimal value, the second the optimal value of the LP relaxation, and the third the computing time (in CPU seconds) needed to solve the LP relaxation. Atamtürk's instances are instead addressed in Tables 7 and 8 . Column "Type of Cuts" gives the type of cuts generated: Gomory mixed-integer cuts (GMI), $k$-cuts [5] for all $k=1,2, \cdots, 50$ (1:50-cuts), and interpolated subadditive cuts when fixing $k=10,20,30,60$. A star indicates an improved bound with respect to GMI. All other columns are self-explanatory. As to knapsack problems (KP), for both the binary and the bounded cases we generated 7 sets of problems, each set consisting of 30 instances with $n$ variables $(n=10,50,100,500,1000,5000,10000) .4$ The corresponding average results are reported in Table 9.

According to the tables, interpolated subadditive cuts do improve the quality of the LP relaxation, but for MIPLIB instances they seldom beat GMI cuts. This negative result confirms the theoretical findings of Dash and Gunluk [7], who showed that interpolated subadditive cuts are dominated by Gomory mixed-integer cuts in a probabilistic sense, as well as the computational experience reported by Cornuejols, Li and Vandenbussche [5] for the subfamily of $k$-cuts. On the other hand, for the hard Atamtürk's multiple-knapsack instances the interpolated cuts allow for a considerable improvement over the GMI bound. As expected, larger values of $k$ produce better bounds, but the separation procedure becomes computationally quite expensive for $k>20$.

For the random single-knapsack problems, the results show that a few interpolated subadditive cuts are able to improve the GMI bound considerably. It should be observed however that, for KP instances, the integrality gap is extremely narrow, so even a very small improvement of the lower bound produces a significant difference in the percentage of gap closed. Comparison with 1:50-cuts shows clearly the negative effect of the interpolation - without interpolation, the family of subadditive cuts would contain all $k$-cuts, hence

\footnotetext{
${ }^{4}$ These problems are very sensitive to the mipgap tolerance parameter used by ILOG CPLEX: in these experiments we used the value $10^{-9}$ for this parameter.
} 
our procedure would guarantee a bound never worse than the 1:50-cut one. This is confirmed by the additional results reported in Table 10, where we addressed KP instances generated as explained above, but with $p_{i}$ and $w_{i}$ uniformly random integers in $[1,100]$ instead of $[1,1000]$ (this guarantees that the ideal $k$ is not larger than 100 , hence it can be handled effectively by our separation procedure; see row "ideal $k$ " in the table). As expected, working with the ideal $k$ produces significantly better results-at least, for our KP instances. A plot of the percentage of closed gap vs. the group-order $k$ is given in Figure 5 for a sample binary KP instance with 50 variables; note that, as expected, the bound growth is not monotone (though for other instances the curve is much more regular, with a saturation starting well before the ideal $k$ ).

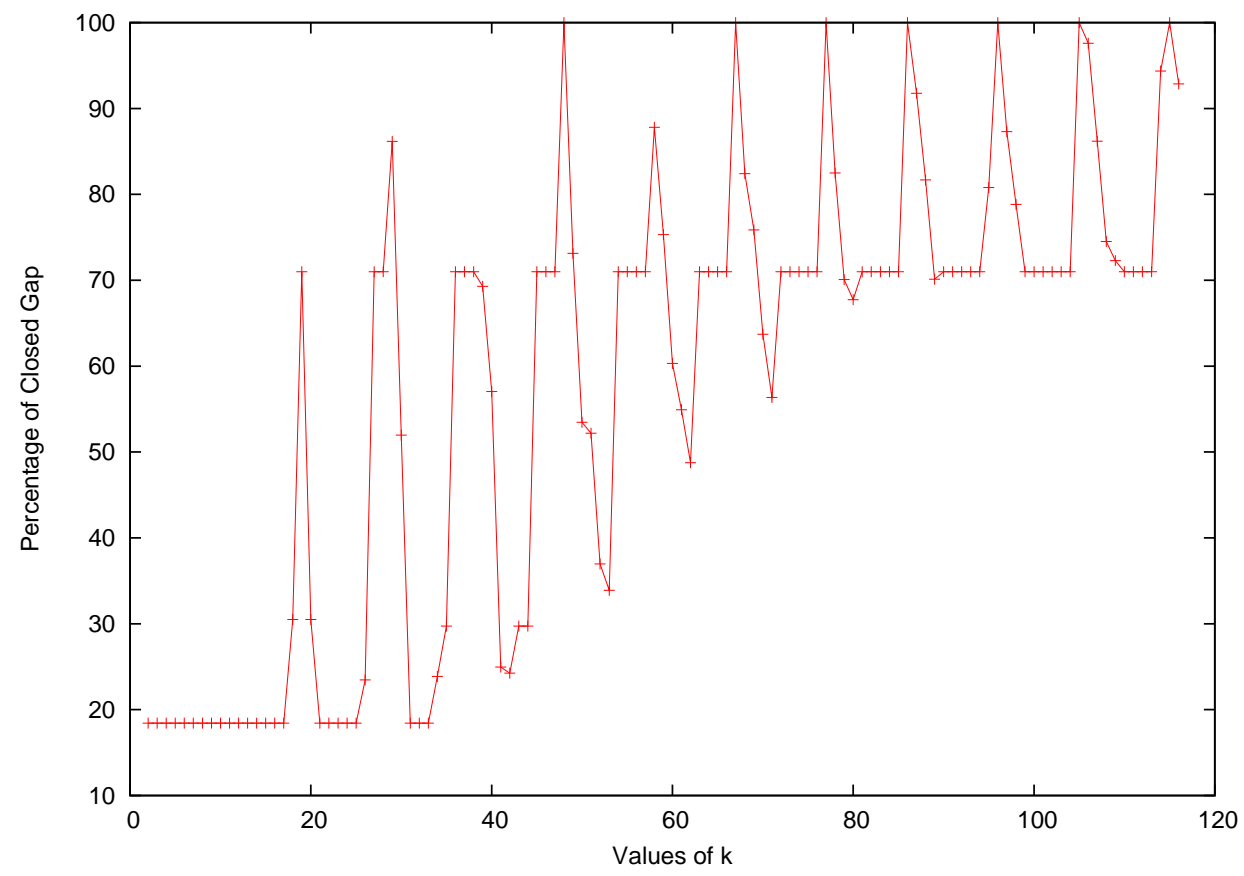

Figure 5: Percentage of closed gap vs. the group-order $k$ for a sample binary $\mathrm{KP}$ instance with 50 variables; the ideal $k$ is 77 in this example

It is unclear at this point whether working with a large $k$ so as to get rid of the interpolation effect, is likely to produce a significant improvement over GMI cuts for a wide class of MIPs. We conjecture that, for problems with a large number of constraints as those in the MIPLIB, the considerable number of GMI cuts read from the optimal tableau is likely to bring the fractional point inside all the group polyhedra associated with the single tableau rows - hence the new fractional point cannot be cut anymore by any subadditive cut (interpolated or not), meaning that the root-node lower bound cannot be improved any further by our approach. If this explanation 
is true, then the research on improved bounds based on mod-1 considerations should concentrate on finding row combinations different from those in the optimal tableau (a topic investigated in a recent paper by Fischetti and Lodi [9]), or has to take into account two or more tableau rows at a same time so as to better approximate the corner polyhedron.

\section{Future directions of work}

Future work on cyclic-group cuts should investigate the following issues.

\section{Integration within a branch-and-cut method}

As already mentioned, an explanation of the good performance of GMI cuts in that these cuts are often able to bring the fractional point inside all the group polyhedra associated with the single tableau rows. However, at a later time, the cutting plane (and branching) process is likely to introduce other constraints that may bring the fractional point outside these group polyhedra, thus triggering our separation procedure to produce new violated subadditive cuts to "move back" the fractional point inside the same group polyhedra-thus hopefully improving the lower bound.

Moreover, the large number of subadditive cuts generated and the small improvement obtained in some cases, would suggest a more conservative policy to better exploit subadditive cuts within a branch-and-cut solution scheme. To be more specific, we believe that a better compromise between lower bound quality and computing time could be reached if one uses first a clever set of non-interpolated subadditive functions to derive quickly an initial set of violated inequalities (including GMI and $k$-cuts), and applies $g$-SEP separation only afterwards. This goes into the direction suggested by Andreello, Caprara and Fischetti [1] for an effective use of easy-to-compute cuts such as GMI and $k$-cuts.

\section{Using higher-rank subadditive cuts}

In our computational experiments we deliberately avoided exploiting the rows of the optimal tableaux obtained after the addition of new cuts, as we were only interested in the rank-1 corner polyhedron associated with the first tableau. In practice, however, one could derive subadditive cuts from the equations of any tableau, just as one can generate several rounds GMI cuts. Practical experience shows that the quality of GMI cuts tends to deteriorate rapidly as new cuts are added, hence one typically avoids their generation after a while. It would be interesting to investigate whether subadditive cuts are also affected by a similar tailing-off phenomenon, the hope being that choosing a cut in the whole family of sudadditive functions (rather than 
choosing only the GMI one) can lead to some improvement.

\section{Replacing a single GMI cut by two subadditive cuts}

In an early stage of our study, we conjectured the effectiveness of GMI cut be due to the fact that it is the deepest ${ }^{5}$ one in the family of subadditive cuts, with respect to the fractional LP solution $x^{*}$ associated with the initial optimal tableau (the one whose rows are stored in our equation pool). Indeed, it is known [4] (and geometrically intuitive) that deep cuts are likely to be the most effective to be used in cutting plane algorithms.

Being the amount of violation for the LP solution $x^{*}$ with respect to any subadditive cut read from the associated tableau a constant, the cut depth actually depends only on the coefficient norm $\|g\|^{2}$, the smaller the norm the deeper the cut.

In order to avoid any dependency on the actual value of the coefficients in the tableau rows, we decided to work on the $T$-space, and computed the deepest cut by solving the quadratic problem $\min \left\{\sum_{i=1}^{k-1} g_{i}^{2}:(10)-\right.$ (12)\}. Surprisingly, we found that the GMI cut (tough quite deep) is not the deepest one, the latter arising when setting $g_{i}=0$ for $i=0, g_{i}=1$ for $i=r$, and $g_{i}=0.5$ otherwise. This produces the cut $\sum_{i=1}^{k-1} t_{i}+t_{r} \geq 2$ saying that any integer $t \in T(k, r)$ has to satisfy the disjunction $\left(t_{r} \geq\right.$ 1) $\bigvee\left(\sum_{i \neq r} t_{i} \geq 2\right)$. The counterpart of the above cut in the $x$-space reads

$$
\sum_{j: \phi\left(\alpha_{j}\right)>0} x_{j}+\sum_{j:\left|\phi\left(\alpha_{j}\right)-\phi(\beta)\right| \leq \epsilon} x_{j} \geq 2
$$

where $\epsilon$ is a very small positive value.

According to our computational experience, however, cut (20) has a poor practical performance. As a matter of fact, its associated subadditive function does not correspond to an extreme point of the $g$-system, hence the corresponding cut does not define a facet of $T(k, r)$. This implies that we can obtain two (and possibly more) facet-defining inequalities for $T(k, r)$ whose convex combination gives the deepest cut (20), a situation illustrated in Figure 6. E.g., for $T(10,5)$ the two extreme solutions $\left(g_{0}, \cdots, g_{9}\right)$ of the $g$-model are given in the rows of the following matrix:

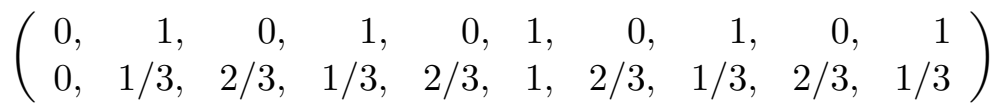

whose combination with weights $1 / 4$ (first row) and $3 / 4$ (second row) produce precisely the deepest-cut function, namely:

$$
(0,1 / 2,1 / 2,1 / 2,1 / 2,1,1 / 2,1 / 2,1 / 2,1 / 2)
$$

It is then natural to investigate the possibility of using the above pair of subadditive cuts instead of (or together with) the usual GMI one, in the

\footnotetext{
${ }^{5}$ The depth of a cut $g^{T} x \geq g_{0}$ with respect to a point $\bar{x}$ is computed as $\left|g^{T} \bar{x}-g_{0}\right| /\|g\|^{2}$
} 


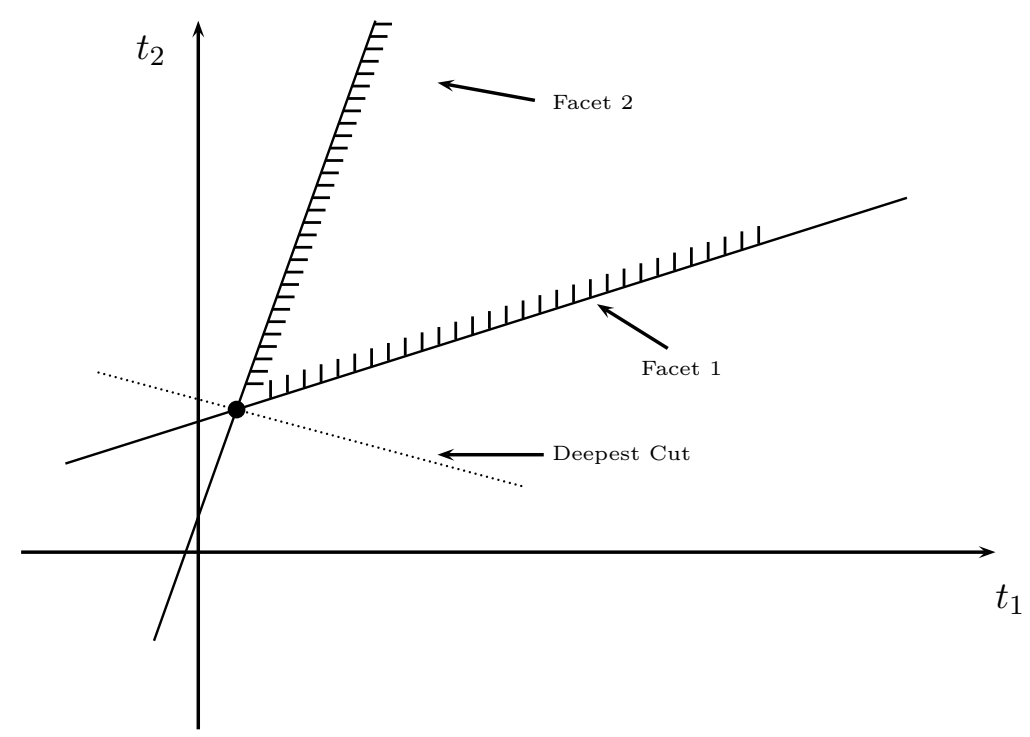

Figure 6: The deepest cut (w.r.t. to the origin) is not a facet of $T(k, r)$, hence it can expressed as the sum of two (or more) such facets

hope that they can "cut from different angles" the factional vertex, hence producing an improved performance without the overhead involved in the solution of the cyclic-group separation problem.

\section{Acknowledgements}

Work supported by MIUR, Italy, and by the EU project ADONET. We thank Andrea Lodi for stimulating discussions about GMI cuts and other subadditive cuts.

\section{References}

[1] G. Andreello, A. Caprara, M. Fischetti, Embedding Cuts in a Branch and Cut Framework: a Computational Study with $\{0,1 / 2\}$-Cuts, to appear in INFORMS Journal on Computing (2006)

[2] A. Atamtürk, http://www.ieor.berkeley.edu/ atamturk/data/ mixed.integer.knapsack/

[3] J. Araoz, R.E. Gomory, E.L. Johnson, and L. Evans, Cyclic group and knapsack facets, Mathematical Programming 96, 377-408 (2003). 
[4] E. Balas, S. Ceria, G. Cornuejols, N. Natraj, Gomory Cuts Revisited, Operations Research Letters 19, 1-9 (1996).

[5] G. Cornuejols, Y. Li and D. Vandenbussche, K-Cuts: A Variation of Gomory Mixed Integer Cuts from the LP Tableau, INFORMS Journal on Computing 15, 385-396 (2003).

[6] S. Dash and O. Gunluk, Valid Inequalities based on Simple MixedInteger Sets, IBM Research Report RC22922, T. J. Watson Research Center, Yorktown Heights, New York (2003).

[7] S. Dash and O. Gunluk, Valid inequalities based on the Interpolation Procedure, IBM Research Report RC22922, T. J. Watson Research Center, Yorktown Heights, New York (2004).

[8] L. Evans, Cyclic Groups and Knapsack Facets with Applications to Cutting Planes, Ph.D. Thesis, Georgia Institute of Technology, Atlanta, Georgia, (2002).

[9] M. Fischetti, A. Lodi, Optimizing over the First Chvátal closure, Integer Programming and Combinatorial Optimization (M. Juenger and V. Kaibel eds.), Lecture Notes in Computer Science 3509, Springer-Verlag Berlin Heidelberg, 12-22 (2005).

[10] R.E. Gomory, Outline of an Algorithm for Integer Solutions to Linear Programs, Bulletin of the AMS 64, 275-278 (1958).

[11] R.E. Gomory, An Algorithm for the Mixed-Integer Problem. Report RM-2597, Rand Corporation (1960).

[12] R.E. Gomory, Some Polyhedra Related to Combinatorial Problems, Journal of Linear Algebra and its Applications 2, 451-558 (1969).

[13] R.E. Gomory and E.L. Johnson, Some Continuous Functions Related to Corner Polyhedra I, Mathematical Programming 3, 23-85 (1972).

[14] R.E. Gomory and E.L. Johnson, Some Continuous Functions Related to Corner Polyhedra II, Mathematical Programming 3, 359-389 (1972).

[15] R.E. Gomory and E.L. Johnson, T-space and Cutting Planes, Mathematical Programming 96, 341-375 (2003).

[16] R.E. Gomory, E. Johnson, and L. Evans, Corner Polyhedra and their connection with cutting planes, Mathematical Programming 96, 321339 (2003).

[17] ILOG Cplex 9.0: User's Manual and Reference Manual, ILOG, S.A., http://www.ilog.com/ (2004). 
[18] ILOG Concert Technology 2.0: User's Manual and Reference Manual, ILOG, S.A., http://www.ilog.com/ (2004).

[19] Letchford A.N. and A. Lodi, Strengthening Chvàtal-Gomory cuts and Gomory fractional cuts, Operations Research Letters 30(2), 74-82, 2002.

[20] M. Koppe, Q. Louveaux, R. Weismantel and L.A. Wolsey, Extended Formulations for Gomory Corner Polyhedra, Discrete Optimization Discrete Optimization 1, 141-165, 2004.

[21] MIPLIB - Mixed Integer Problem Library 2003, http://miplib.zib.de (2003)

[22] G.L. Nemhauser and L.A. Wolsey, Integer and Combinatorial Optimization, Wiley, New York (1988). 
Table 1: MIPLIB mixed integer problems (part 1).

\begin{tabular}{|c|c|c|c|c|c|c|}
\hline Problem & $\begin{array}{l}\text { Type of } \\
\text { Cuts }\end{array}$ & Final LB & $\begin{array}{c}\text { Closed } \\
\text { Gap } \\
(\%)\end{array}$ & $\begin{array}{c}\text { Separation } \\
\text { Time } \\
\text { (seconds) }\end{array}$ & $\begin{array}{c}\text { Total } \\
\text { Time } \\
\text { (seconds) }\end{array}$ & $\begin{array}{c}\text { Number of } \\
\text { Cuts }\end{array}$ \\
\hline & 1:50-cuts & $\overline{9924.00}$ & 100.00 & 10.04 & 14.85 & $\overline{275}$ \\
\hline 10teams & GMI & 924.00 & 100.00 & 0.20 & 2.95 & 162 \\
\hline 924.00 & $\mathrm{~K}=10$ & 924.00 & 100.00 & 0.33 & 2.73 & 162 \\
\hline 917.00 & $\mathrm{~K}=20$ & 924.00 & 100.00 & 0.53 & 3.04 & 162 \\
\hline \multirow[t]{2}{*}{0.16} & $\mathrm{~K}=30$ & 924.00 & 100.00 & 1.06 & 3.69 & 162 \\
\hline & $\mathrm{K}=60$ & 924.00 & 100.00 & 12.07 & 14.66 & 162 \\
\hline & $1: 50$-cuts & 1002.66 & 11.15 & 0.96 & 1.48 & 500 \\
\hline aflow30a & GMI & 1002.66 & 11.15 & 0.01 & 0.05 & 31 \\
\hline 1158.00 & $\mathrm{~K}=10$ & 1001.55 & 10.51 & 0.07 & 0.17 & 56 \\
\hline 983.17 & $\mathrm{~K}=20$ & 1002.21 & 10.89 & 0.22 & 0.38 & 64 \\
\hline \multirow[t]{2}{*}{0.01} & $\mathrm{~K}=30$ & 1002.35 & 10.97 & 0.83 & 0.99 & 72 \\
\hline & $\mathrm{K}=60$ & 1002.54 & 11.08 & 12.30 & 12.45 & 72 \\
\hline & $1: 50$-cuts $\star$ & 1014.56 & 5.48 & 3.91 & 5.75 & 379 \\
\hline aflow $40 b$ & GMI & 1014.56 & 5.48 & 0.06 & 0.39 & 38 \\
\hline 1168.00 & $\mathrm{~K}=10 \star$ & 1014.27 & 5.30 & 0.19 & 0.78 & 57 \\
\hline 1005.66 & $\mathrm{~K}=20 \star$ & 1014.46 & 5.42 & 0.43 & 1.29 & 64 \\
\hline \multirow[t]{2}{*}{0.11} & $\mathrm{~K}=30$ 夫 & 1014.50 & 5.44 & 1.05 & 1.84 & 63 \\
\hline & $\mathrm{K}=60 \star$ & 1014.51 & 5.45 & 17.81 & 18.60 & 66 \\
\hline & $1: 50$-cuts & 872157.51 & 60.43 & 0.32 & 0.42 & 600 \\
\hline bell3a & GMI & 872157.51 & 60.43 & 0.00 & 0.00 & 50 \\
\hline 878430.32 & $\mathrm{~K}=10$ & 872015.80 & 59.53 & 0.04 & 0.04 & 83 \\
\hline 862578.64 & $\mathrm{~K}=20$ & 872092.20 & 60.02 & 0.22 & 0.23 & 93 \\
\hline \multirow[t]{2}{*}{0.00} & $\mathrm{~K}=30$ & 872099.44 & 60.06 & 0.59 & 0.62 & 96 \\
\hline & $\mathrm{K}=60$ & 872140.08 & 60.32 & 11.27 & 11.29 & 93 \\
\hline & $1: 50$-cuts & 8660422.46 & 14.53 & 0.21 & 0.27 & 464 \\
\hline bell5 & GMI & 8660422.46 & 14.53 & 0.01 & 0.01 & 40 \\
\hline 8966406.49 & $K=10$ & 8654669.96 & 12.92 & 0.03 & 0.04 & 59 \\
\hline 8608417.95 & $\mathrm{~K}=20$ & 8657274.83 & 13.65 & 0.13 & 0.14 & 60 \\
\hline \multirow[t]{2}{*}{0.00} & $\mathrm{~K}=30$ & 8658662.54 & 14.04 & 0.40 & 0.41 & 59 \\
\hline & $\mathrm{K}=60 \star$ & 8661152.43 & 14.73 & 12.73 & 12.76 & 77 \\
\hline & $1: 50$-cuts & 6.92 & 0.00 & 0.06 & 0.06 & 0 \\
\hline blend2 & GMI & 6.92 & 0.00 & 0.00 & 0.00 & 0 \\
\hline 7.60 & $\mathrm{~K}=10$ & 6.92 & 0.00 & 0.01 & 0.01 & 0 \\
\hline 6.92 & $\mathrm{~K}=20$ & 6.92 & 0.00 & 0.01 & 0.01 & 0 \\
\hline \multirow{2}{*}{0.01} & $\mathrm{~K}=30$ & 6.92 & 0.00 & 0.02 & 0.02 & 0 \\
\hline & $\mathrm{K}=60$ & 6.92 & 0.00 & 0.88 & 0.88 & 0 \\
\hline & $1: 50$-cuts & 62.69 & 1.74 & 5.52 & 8.17 & 224 \\
\hline danoint & GMI & 62.69 & 1.74 & 0.04 & 0.67 & 52 \\
\hline 65.67 & $\mathrm{~K}=10$ & 62.65 & 0.39 & 0.25 & 1.16 & 74 \\
\hline 62.64 & $\mathrm{~K}=20$ & 62.65 & 0.55 & 1.00 & 2.01 & 81 \\
\hline \multirow{2}{*}{$\begin{array}{l}0.21 \\
0.21\end{array}$} & $\mathrm{~K}=30$ & 62.66 & 0.68 & 3.55 & 4.55 & 80 \\
\hline & $\mathrm{K}=60$ & 62.67 & 0.96 & 71.12 & 72.26 & 82 \\
\hline & $1: 50$-cuts $\star$ & 291699.72 & 54.28 & 2.34 & 2.79 & 646 \\
\hline fiber & GMI & 279391.25 & 49.35 & 0.03 & 0.05 & 46 \\
\hline 405935.18 & $\mathrm{~K}=10 \star$ & 268704.27 & 45.08 & 0.12 & 0.19 & 75 \\
\hline 156082.52 & 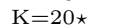 & 277434.30 & 48.57 & 0.39 & 0.49 & 103 \\
\hline \multirow[t]{2}{*}{0.01} & $\mathrm{~K}=30 \star$ & 282035.42 & 50.41 & 1.43 & 1.58 & 133 \\
\hline & $\mathrm{K}=60 \star$ & 285955.48 & 51.98 & 33.19 & 33.29 & 119 \\
\hline & $1: 50$-cuts & 1171213.72 & 11.74 & 0.00 & 0.03 & 204 \\
\hline flugpl & GMI & 1171213.72 & 11.74 & 0.00 & 0.00 & 10 \\
\hline 1201500.00 & $\mathrm{~K}=10$ & 1171004.98 & 11.13 & 0.01 & 0.01 & 19 \\
\hline 1167185.73 & $\mathrm{~K}=20$ & 1171142.46 & 11.53 & 0.04 & 0.04 & 20 \\
\hline \multirow[t]{2}{*}{0.00} & $\mathrm{~K}=30$ & 1171193.55 & 11.68 & 0.14 & 0.14 & 18 \\
\hline & $\mathrm{K}=60$ & 1171197.64 & 11.69 & 3.85 & 3.86 & 19 \\
\hline & $1: 50$-cuts & 112239.39 & 59.77 & 3.04 & 3.77 & 800 \\
\hline gen & GMI & 112239.39 & 59.77 & 0.03 & 0.14 & 45 \\
\hline 112313.00 & $K=10$ & 112216.67 & 47.35 & 0.17 & 0.33 & 71 \\
\hline 112130.04 & $\mathrm{~K}=20$ & 112222.13 & 50.34 & 0.50 & 0.68 & 84 \\
\hline 0.05 & $\mathrm{~K}=30$ & 112228.97 & 54.07 & 2.96 & 3.21 & 76 \\
\hline & $\mathrm{K}=60$ & 112239.37 & 59.75 & 55.87 & 56.20 & 94 \\
\hline & $1: 50$-cuts & 25568014.40 & 30.17 & 21.37 & 24.11 & 1816 \\
\hline gesa2 & GMI & 25568014.40 & 30.17 & 0.18 & 0.35 & 91 \\
\hline 25779856.37 & $\mathrm{~K}=10$ & 25565828.17 & 29.45 & 0.56 & 0.86 & 178 \\
\hline 25476489.68 & $\mathrm{~K}=20$ & 25566860.50 & 29.79 & 1.63 & 2.04 & 206 \\
\hline 0.09 & $\mathrm{~K}=30$ & 25567378.76 & 29.96 & 5.32 & 5.83 & 206 \\
\hline & $\mathrm{K}=60$ & 25567569.09 & 30.02 & 100.04 & 100.60 & 204 \\
\hline & $1: 50$-cuts $\star$ & 25568387.00 & 30.29 & 24.88 & 27.32 & 2041 \\
\hline & GMI & 25567400.54 & 29.97 & 0.26 & 0.41 & 121 \\
\hline 25779856.37 & $\mathrm{~K}=10 \star$ & 25565930.89 & 29.48 & 0.74 & 1.04 & 218 \\
\hline 25476489.68 & 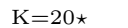 & 25567122.07 & 29.88 & 1.72 & 2.09 & 251 \\
\hline 0.05 & $\mathrm{~K}=30 \star$ & 25567712.56 & 30.07 & 5.50 & 5.94 & 243 \\
\hline & $\mathrm{K}=60 \star$ & 25568060.92 & 30.19 & 88.02 & 88.53 & 258 \\
\hline
\end{tabular}


Table 2: MIPLIB mixed integer problems (part 2).

\begin{tabular}{|c|c|c|c|c|c|c|}
\hline Problem & $\begin{array}{l}\text { Type of } \\
\text { Cuts }\end{array}$ & Final LB & $\begin{array}{c}\text { Closed } \\
\text { Gap } \\
(\%)\end{array}$ & $\begin{array}{c}\text { Separation } \\
\text { Time } \\
\text { (seconds) }\end{array}$ & $\begin{array}{c}\text { Total } \\
\text { Time } \\
\text { (seconds) }\end{array}$ & $\begin{array}{c}\text { Number of } \\
\text { Cuts }\end{array}$ \\
\hline & 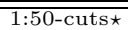 & 19092.03 & $\overline{73.09}$ & $\overline{00.11}$ & $\overline{00.18}$ & $\overline{\overline{410}}$ \\
\hline gt2 & GMI & 18998.90 & 71.88 & 0.00 & 0.00 & 12 \\
\hline 21166.00 & $K=10 \star$ & 15241.29 & 23.11 & 0.01 & 0.01 & 16 \\
\hline 13460.23 & 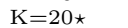 & 15241.29 & 23.11 & 0.03 & 0.04 & 18 \\
\hline \multirow[t]{2}{*}{0.00} & $\mathrm{~K}=30 \star$ & 15463.52 & 26.00 & 0.17 & 0.19 & 23 \\
\hline & $K=60 \star$ & 17986.99 & 58.74 & 4.80 & 4.81 & 23 \\
\hline & $1: 50$-cuts & 0.00 & 0.00 & 0.00 & 0.01 & 200 \\
\hline markshare1 & GMI & 0.00 & 0.00 & 0.00 & 0.00 & 6 \\
\hline 1.00 & $\mathrm{~K}=10$ & 0.00 & 0.00 & 0.00 & 0.00 & 6 \\
\hline 0.00 & $\mathrm{~K}=20$ & 0.00 & 0.00 & 0.02 & 0.02 & 7 \\
\hline \multirow[t]{2}{*}{0.00} & $\mathrm{~K}=30$ & 0.00 & 0.00 & 0.04 & 0.04 & 6 \\
\hline & $\mathrm{K}=60$ & 0.00 & 0.00 & 0.60 & 0.60 & 6 \\
\hline & $1: 50$-cuts & 0.00 & 0.00 & 0.01 & 0.02 & 200 \\
\hline markshare2 & GMI & 0.00 & 0.00 & 0.00 & 0.00 & 7 \\
\hline 1.00 & $\mathrm{~K}=10$ & 0.00 & 0.00 & 0.00 & 0.00 & 7 \\
\hline 0.00 & $\mathrm{~K}=20$ & 0.00 & 0.00 & 0.01 & 0.01 & 7 \\
\hline \multirow[t]{2}{*}{0.00} & $\mathrm{~K}=30$ & 0.00 & 0.00 & 0.05 & 0.05 & 7 \\
\hline & $\mathrm{K}=60$ & 0.00 & 0.00 & 0.55 & 0.55 & 7 \\
\hline & $1: 50$-cuts $\star$ & 10578.59 & 7.27 & 0.13 & 0.44 & 230 \\
\hline $\operatorname{mas} 74$ & GMI & 10570.72 & 6.67 & 0.00 & 0.01 & 12 \\
\hline 11801.20 & $\mathrm{~K}=10 \star$ & 10570.94 & 6.69 & 0.01 & 0.04 & 33 \\
\hline 10482.80 & $\mathrm{~K}=20 \star$ & 10576.54 & 7.11 & 0.12 & 0.15 & 44 \\
\hline \multirow[t]{2}{*}{0.01} & $\mathrm{~K}=30 \star$ & 10581.80 & 7.51 & 0.83 & 0.90 & 71 \\
\hline & $\mathrm{K}=60 \star$ & 10585.87 & 7.82 & 27.15 & 27.23 & 79 \\
\hline & $1: 50$-cuts $\star$ & 38971.87 & 7.02 & 0.07 & 0.30 & 221 \\
\hline mas76 & GMI & 38965.29 & 6.42 & 0.00 & 0.01 & 11 \\
\hline 40005.10 & $K=10 \star$ & 38968.36 & 6.70 & 0.02 & 0.04 & 25 \\
\hline 38893.90 & $\mathrm{~K}=20 \star$ & 38972.76 & 7.10 & 0.12 & 0.14 & 43 \\
\hline \multirow[t]{2}{*}{0.00} & $\mathrm{~K}=30 \star$ & 38975.64 & 7.36 & 0.49 & 0.53 & 34 \\
\hline & $K=60 \star$ & 38977.76 & 7.55 & 19.21 & 19.26 & 52 \\
\hline & $1: 50$-cuts $\star$ & -608.67 & 6.62 & 82.83 & 88.59 & 950 \\
\hline mkc & GMI & -609.41 & 5.09 & 0.69 & 1.16 & 142 \\
\hline-563.85 & $\mathrm{~K}=10 \star$ & -609.32 & 5.27 & 4.98 & 6.91 & 367 \\
\hline-611.85 & $K=20 \star$ & -609.32 & 5.27 & 8.56 & 11.19 & 463 \\
\hline \multirow{2}{*}{0.09} & $K=30 \star$ & -609.08 & 5.76 & 16.49 & 20.09 & 600 \\
\hline & $\mathrm{K}=60 \star$ & -608.92 & 6.11 & 408.84 & 416.52 & 958 \\
\hline & $1: 50-\operatorname{cuts} \star$ & 294.29 & 20.92 & 0.05 & 0.15 & 200 \\
\hline $\bmod 008$ & GMI & 294.29 & 20.88 & 0.00 & 0.01 & 5 \\
\hline 307.00 & $\mathrm{~K}=10 \star$ & 293.95 & 18.80 & 0.00 & 0.00 & 8 \\
\hline 290.93 & $K=20 \star$ & 294.17 & 20.18 & 0.02 & 0.03 & 8 \\
\hline \multirow[t]{2}{*}{$\begin{array}{l}290.90 \\
0.00\end{array}$} & $\mathrm{~K}=30$ * & 294.28 & 20.81 & 0.06 & 0.06 & 8 \\
\hline & $\mathrm{K}=60 \star$ & 294.28 & 20.83 & 2.29 & 2.30 & 9 \\
\hline & $1: 50$-cuts & 20484452.96 & 17.28 & 0.92 & 1.23 & 800 \\
\hline modglob & GMI & 20484452.96 & 17.28 & 0.01 & 0.03 & 30 \\
\hline 20740508.00 & $\mathrm{~K}=10$ & 20472029.03 & 13.27 & 0.06 & 0.09 & 52 \\
\hline 20430947.62 & $\mathrm{~K}=20$ & 20478791.33 & 15.46 & 0.23 & 0.29 & 50 \\
\hline \multirow[t]{2}{*}{0.01} & $\mathrm{~K}=30$ & 20481755.99 & 16.41 & 0.85 & 0.92 & 54 \\
\hline & $\mathrm{K}=60$ & 20482796.42 & 16.75 & 24.97 & 25.03 & 55 \\
\hline & $1: 50$-cuts & 31.16 & 7.07 & 2041.36 & 2439.95 & 819 \\
\hline net12 & GMI & 31.16 & 7.07 & 33.49 & 66.35 & 296 \\
\hline 214.00 & $\mathrm{~K}=10$ & 30.31 & 6.64 & 29.38 & 75.80 & 457 \\
\hline 17.25 & $\mathrm{~K}=20$ & 30.69 & 6.83 & 39.63 & 86.34 & 462 \\
\hline \multirow{2}{*}{14.53} & $\mathrm{~K}=30$ & 31.02 & 7.00 & 47.41 & 92.83 & 433 \\
\hline & $K=60$ & 31.10 & 7.04 & 268.56 & 312.26 & 477 \\
\hline & $1: 50$-cuts $\star$ & -19.23 & 19.74 & 0.70 & 1.34 & 349 \\
\hline opt1217 & GMI & -19.23 & 19.72 & 0.01 & 0.04 & 28 \\
\hline-16.00 & $K=10 \star$ & -19.26 & 18.85 & 0.09 & 0.15 & 53 \\
\hline-20.02 & $K=20 \star$ & -19.25 & 19.11 & 0.16 & 0.23 & 55 \\
\hline 0.01 & $\mathrm{~K}=30 \star$ & -19.23 & 19.67 & 0.57 & 0.71 & 68 \\
\hline & $\mathrm{K}=60 \star$ & -19.23 & 19.68 & 12.43 & 12.57 & 75 \\
\hline & $1: 50$-cuts & 0.00 & 0.00 & 0.02 & 0.04 & 200 \\
\hline pk1 & GMI & 0.00 & 0.00 & 0.00 & 0.01 & 15 \\
\hline 11.00 & $\mathrm{~K}=10$ & 0.00 & 0.00 & 0.01 & 0.01 & 15 \\
\hline 0.00 & $\mathrm{~K}=20$ & 0.00 & 0.00 & 0.02 & 0.03 & 15 \\
\hline 0.01 & $\mathrm{~K}=30$ & 0.00 & 0.00 & 0.07 & 0.07 & 15 \\
\hline & $\mathrm{K}=60$ & 0.00 & 0.00 & 1.25 & 1.25 & 15 \\
\hline & $1: 50$-cuts & 5151.26 & 52.22 & 0.41 & 0.75 & 1846 \\
\hline & GMI & 5151.26 & 52.22 & 0.00 & 0.01 & 53 \\
\hline 7350.00 & $\mathrm{~K}=10$ & 5073.19 & 50.52 & 0.06 & 0.08 & 92 \\
\hline 2748.35 & $\mathrm{~K}=20$ & 5115.98 & 51.45 & 0.16 & 0.18 & 102 \\
\hline 0.00 & $\mathrm{~K}=30$ & 5124.13 & 51.63 & 0.57 & 0.61 & 111 \\
\hline & $\mathrm{K}=60$ & 5144.84 & 52.08 & 10.61 & 10.63 & 110 \\
\hline
\end{tabular}


Table 3: MIPLIB mixed integer problems (part 3).

\begin{tabular}{|c|c|c|c|c|c|c|}
\hline Problem & $\begin{array}{l}\text { Type of } \\
\text { Cuts }\end{array}$ & Final LB & $\begin{array}{c}\text { Closed } \\
\text { Gap } \\
(\%)\end{array}$ & $\begin{array}{c}\text { Separation } \\
\text { Time } \\
\text { (seconds) } \\
\end{array}$ & $\begin{array}{c}\text { Total } \\
\text { Time } \\
\text { (seconds) } \\
\end{array}$ & $\begin{array}{c}\text { Number of } \\
\text { Cuts }\end{array}$ \\
\hline & $1: 50$-cuts $\star$ & 14447.39 & 9.87 & 3.84 & 6.72 & $\overline{374}$ \\
\hline qnet1 & GMI & 14445.72 & 9.78 & 0.09 & 0.26 & 55 \\
\hline 16029.69 & $K=10 \star$ & 14446.24 & 9.80 & 0.22 & 0.49 & 59 \\
\hline 14274.10 & $\mathrm{~K}=20 \star \star$ & 14446.24 & 9.80 & 0.38 & 0.64 & 63 \\
\hline \multirow[t]{2}{*}{0.05} & $\mathrm{~K}=30 \star$ & 14446.24 & 9.80 & 1.01 & 1.32 & 69 \\
\hline & $\mathrm{K}=60 \star \star$ & 14447.10 & 9.85 & 23.74 & 24.09 & 75 \\
\hline & $1: 50$-cuts $\star$ & 13748.85 & 42.02 & 1.07 & 1.99 & 400 \\
\hline qnet1_o & GMI & 13739.62 & 41.79 & 0.03 & 0.07 & 11 \\
\hline 16029.69 & $\mathrm{~K}=10 \star$ & 13686.09 & 40.43 & 0.09 & 0.18 & 22 \\
\hline 12095.57 & $K=20 \star$ & 13714.81 & 41.16 & 0.21 & 0.33 & 29 \\
\hline \multirow{2}{*}{0.02} & $\mathrm{~K}=30 \star$ & 13724.55 & 41.41 & 0.49 & 0.61 & 31 \\
\hline & $\mathrm{K}=60 \star \star$ & 13738.61 & 41.76 & 14.90 & 15.03 & 30 \\
\hline & $1: 50$-cuts & 29046932.15 & 15.53 & 46.90 & 64.34 & 550 \\
\hline rentacar & GMI & 29046932.15 & 15.53 & 0.78 & 6.82 & 22 \\
\hline 30356761.00 & $\mathrm{~K}=10$ & 28806137.64 & 0.00 & 1.51 & 7.47 & 32 \\
\hline 28806137.64 & $\mathrm{~K}=20$ & 28806137.64 & 0.00 & 3.01 & 7.72 & 31 \\
\hline \multirow[t]{2}{*}{7.19} & $\mathrm{~K}=30$ & 28806137.64 & 0.00 & 9.91 & 15.91 & 35 \\
\hline & $\mathrm{K}=60$ & 28877221.50 & 4.58 & 150.90 & 159.31 & 39 \\
\hline & $1: 50$-cuts & 40830.12 & 39.16 & 6.35 & 8.42 & 4266 \\
\hline set1ch & GMI & 40830.12 & 39.16 & 0.02 & 0.08 & 138 \\
\hline 54537.80 & $K=10$ & 38975.16 & 30.93 & 0.12 & 0.22 & 258 \\
\hline 32007.73 & $\mathrm{~K}=20$ & 40274.48 & 36.69 & 0.38 & 0.50 & 263 \\
\hline \multirow[t]{2}{*}{0.00} & $\mathrm{~K}=30$ & 40622.20 & 38.24 & 1.40 & 1.53 & 287 \\
\hline & $\mathrm{K}=60$ & 40776.80 & 38.92 & 37.04 & 37.17 & 294 \\
\hline & $1: 50$-cuts & 201721.28 & 23.51 & 1.53 & 1.86 & 1188 \\
\hline timtab1 & GMI & 201721.28 & 23.51 & 0.01 & 0.03 & 136 \\
\hline 764772.00 & $\mathrm{~K}=10$ & 199777.70 & 23.24 & 0.13 & 0.19 & 194 \\
\hline 28694.00 & $\mathrm{~K}=20$ & 200979.95 & 23.41 & 0.47 & 0.54 & 218 \\
\hline \multirow[t]{2}{*}{0.00} & $\mathrm{~K}=30$ & 201158.22 & 23.43 & 1.76 & 1.82 & 244 \\
\hline & $\mathrm{K}=60$ & 201721.28 & 23.51 & 25.59 & 25.64 & 254 \\
\hline & $1: 50$-cuts & 84359.79 & 60.27 & 76.98 & 90.45 & 13262 \\
\hline $\operatorname{tr} 12-30$ & GMI & 84359.79 & 60.27 & 0.13 & 0.36 & 348 \\
\hline 130596.00 & $\mathrm{~K}=10$ & 73768.19 & 51.17 & 0.36 & 0.67 & 550 \\
\hline 14210.43 & $\mathrm{~K}=20$ & 80578.11 & 57.02 & 0.87 & 1.42 & 806 \\
\hline \multirow{2}{*}{0.01} & $\mathrm{~K}=30$ & 83103.48 & 59.19 & 3.45 & 4.02 & 733 \\
\hline & $\mathrm{K}=60$ & 83817.48 & 59.81 & 72.54 & 73.25 & 771 \\
\hline & $1: 50$-cuts & 15.86 & 9.70 & 0.27 & 0.30 & 118 \\
\hline vpm1 & GMI & 15.86 & 9.70 & 0.00 & 0.00 & 7 \\
\hline 20.00 & $\mathrm{~K}=10$ & 15.81 & 8.66 & 0.04 & 0.06 & 18 \\
\hline 15.42 & $\mathrm{~K}=20$ & 15.86 & 9.70 & 0.09 & 0.09 & 11 \\
\hline \multirow[t]{2}{*}{0.00} & $\mathrm{~K}=30$ & 15.85 & 9.37 & 0.37 & 0.38 & 15 \\
\hline & $\mathrm{K}=60$ & 15.86 & 9.70 & 3.90 & 3.92 & 11 \\
\hline & $1: 50$-cuts & 10.36 & 12.15 & 0.47 & 0.60 & 390 \\
\hline vpm2 & GMI & 10.36 & 12.15 & 0.01 & 0.02 & 20 \\
\hline 13.75 & $\mathrm{~K}=10$ & 10.35 & 11.82 & 0.05 & 0.07 & 27 \\
\hline 9.89 & $\mathrm{~K}=20$ & 10.35 & 12.05 & 0.23 & 0.25 & 33 \\
\hline \multirow[t]{2}{*}{0.01} & $\mathrm{~K}=30$ & 10.35 & 12.06 & 0.85 & 0.88 & 36 \\
\hline & $\mathrm{K}=60$ & 10.36 & 12.11 & 19.28 & 19.33 & 42 \\
\hline & $1: 50$-cuts & 390.10 & 57.47 & 0.25 & 0.46 & 1588 \\
\hline egout & GMI & 390.10 & 57.47 & 0.01 & 0.02 & 40 \\
\hline 568.10 & $\mathrm{~K}=10$ & 242.52 & 22.20 & 0.03 & 0.04 & 60 \\
\hline 149.59 & $\mathrm{~K}=20$ & 292.92 & 34.25 & 0.13 & 0.15 & 69 \\
\hline \multirow[t]{2}{*}{0.00} & $\mathrm{~K}=30$ & 304.87 & 37.10 & 0.59 & 0.60 & 75 \\
\hline & $\mathrm{K}=60$ & 319.41 & 40.58 & 14.32 & 14.33 & 71 \\
\hline & $1: 50$-cuts & 1497.17 & 10.65 & 2.76 & 3.89 & 2349 \\
\hline fixnet6 & GMI & 1497.17 & 10.65 & 0.01 & 0.07 & 60 \\
\hline 3983.00 & $\mathrm{~K}=10$ & 1470.33 & 9.69 & 0.07 & 0.14 & 91 \\
\hline 1200.88 & $\mathrm{~K}=20$ & 1478.40 & 9.97 & 0.09 & 0.14 & 92 \\
\hline 0.00 & $\mathrm{~K}=30$ & 1483.45 & 10.16 & 0.28 & 0.32 & 76 \\
\hline & $\mathrm{K}=60$ & 1493.53 & 10.52 & 7.89 & 7.96 & 91 \\
\hline & $1: 50$-cuts & 31.16 & 7.07 & 2041.36 & 2439.95 & 819 \\
\hline net12 & GMI & 31.16 & 7.07 & 33.49 & 66.35 & 296 \\
\hline 214.00 & $\mathrm{~K}=10$ & 30.31 & 6.64 & 29.38 & 75.80 & 457 \\
\hline 17.25 & $\mathrm{~K}=20$ & 30.69 & 6.83 & 39.63 & 86.34 & 462 \\
\hline 14.53 & $\mathrm{~K}=30$ & 31.02 & 7.00 & 47.41 & 92.83 & 433 \\
\hline & $\mathrm{K}=60$ & 31.10 & 7.04 & 268.56 & 312.26 & 477 \\
\hline & $1: 50$-cuts & -43.00 & 0.00 & 0.56 & 0.68 & 666 \\
\hline noswot & GMI & -43.00 & 0.00 & 0.00 & 0.02 & 48 \\
\hline-41.00 & $\mathrm{~K}=10$ & -43.00 & 0.00 & 0.08 & 0.12 & 63 \\
\hline-43.00 & $\mathrm{~K}=20$ & -43.00 & 0.00 & 0.18 & 0.20 & 54 \\
\hline 0.00 & $\mathrm{~K}=30$ & -43.00 & 0.00 & 1.24 & 1.27 & 58 \\
\hline & $\mathrm{K}=60$ & -43.00 & 0.00 & 16.27 & 16.28 & 56 \\
\hline
\end{tabular}


Table 4: MIPLIB mixed integer problems (part 4).

\begin{tabular}{|c|c|c|c|c|c|c|}
\hline Problem & $\begin{array}{l}\text { Type of } \\
\text { Cuts }\end{array}$ & Final LB & $\begin{array}{c}\text { Closed } \\
\text { Gap } \\
(\%)\end{array}$ & $\begin{array}{c}\text { Separation } \\
\text { Time } \\
\text { (seconds) }\end{array}$ & $\begin{array}{c}\text { Total } \\
\text { Time } \\
\text { (seconds) }\end{array}$ & $\begin{array}{c}\text { Number of } \\
\text { Cuts }\end{array}$ \\
\hline & $1: 50$-cuts & 52.26 & 10.37 & 0.06 & 0.10 & 327 \\
\hline rgn & GMI & 52.26 & 10.37 & 0.00 & 0.00 & 16 \\
\hline 82.20 & $\mathrm{~K}=10$ & 51.66 & 8.56 & 0.03 & 0.05 & 27 \\
\hline 48.80 & $K=20$ & 52.00 & 9.59 & 0.05 & 0.06 & 28 \\
\hline \multirow[t]{2}{*}{0.00} & $\mathrm{~K}=30$ & 52.17 & 10.08 & 0.24 & 0.27 & 32 \\
\hline & $K=60$ & 52.17 & 10.08 & 4.75 & 4.77 & 28 \\
\hline & $1: 50$-cuts & 6066.01 & 31.32 & 0.66 & 0.98 & 884 \\
\hline pp08aCUTS & GMI & 6066.01 & 31.32 & 0.00 & 0.03 & 46 \\
\hline 7350.00 & $\mathrm{~K}=10$ & 6043.49 & 30.11 & 0.06 & 0.11 & 72 \\
\hline 5480.61 & $K=20$ & 6059.97 & 30.99 & 0.28 & 0.31 & 76 \\
\hline \multirow[t]{2}{*}{0.01} & $\mathrm{~K}=30$ & 6059.67 & 30.98 & 1.12 & 1.19 & 91 \\
\hline & $\mathrm{K}=60$ & 6063.23 & 31.17 & 28.15 & 28.20 & 92 \\
\hline & $1: 50$-cuts & -924.23 & 0.93 & 6.35 & 11.15 & 410 \\
\hline qiu & GMI & -924.23 & 0.93 & 0.07 & 0.73 & 36 \\
\hline-132.87 & $\mathrm{~K}=10$ & -924.97 & 0.84 & 0.24 & 1.16 & 59 \\
\hline-931.64 & $\mathrm{~K}=20$ & -924.57 & 0.89 & 0.63 & 1.52 & 59 \\
\hline \multirow[t]{2}{*}{0.21} & $\mathrm{~K}=30$ & -924.44 & 0.90 & 2.33 & 3.43 & 60 \\
\hline & $K=60$ & -924.44 & 0.90 & 55.77 & 56.95 & 71 \\
\hline & 1:50-cuts & 27908501.37 & 47.56 & 14.99 & 16.99 & 1550 \\
\hline gesa3 & GMI & 27908501.37 & 47.56 & 0.19 & 0.41 & 100 \\
\hline 27991042.65 & $\mathrm{~K}=10$ & 27906378.34 & 46.21 & 0.62 & 0.93 & 151 \\
\hline 27833632.45 & $\mathrm{~K}=20$ & 27907466.03 & 46.91 & 1.40 & 1.76 & 183 \\
\hline \multirow[t]{2}{*}{0.10} & $\mathrm{~K}=30$ & 27908302.96 & 47.44 & 5.61 & 6.07 & 203 \\
\hline & $\mathrm{K}=60$ & 27908406.33 & 47.50 & 108.63 & 109.18 & 200 \\
\hline & $1: 50$-cuts & 27928910.08 & 60.53 & 19.59 & 21.30 & 1580 \\
\hline gesa3_o & GMI & 27928910.08 & 60.53 & 0.30 & 0.50 & 145 \\
\hline 27991042.65 & $\mathrm{~K}=10$ & 27925661.11 & 58.46 & 0.82 & 1.14 & 216 \\
\hline 27833632.45 & $\mathrm{~K}=20$ & 27927682.03 & 59.75 & 1.86 & 2.28 & 256 \\
\hline \multirow[t]{2}{*}{0.08} & $\mathrm{~K}=30$ & 27928467.67 & 60.25 & 5.18 & 5.75 & 303 \\
\hline & $\mathrm{K}=60$ & 27928634.90 & 60.35 & 95.07 & 95.67 & 282 \\
\hline
\end{tabular}


Table 5: MIPLIB pure integer problems (part 1).

\begin{tabular}{|c|c|c|c|c|c|c|}
\hline Problem & $\begin{array}{l}\text { Type of } \\
\text { Cuts }\end{array}$ & Final LB & $\begin{array}{c}\text { Closed } \\
\text { Gap } \\
(\%) \\
\end{array}$ & $\begin{array}{c}\text { Separation } \\
\text { Time } \\
\text { (seconds) } \\
\end{array}$ & $\begin{array}{c}\text { Total } \\
\text { Time } \\
\text { (seconds) } \\
\end{array}$ & $\begin{array}{c}\text { Number of } \\
\text { Cuts }\end{array}$ \\
\hline & 1:50-cuts & 340160.00 & 100.00 & 0.90 & 5.03 & 200 \\
\hline air03 & GMI & 340160.00 & 100.00 & 0.12 & 1.03 & 36 \\
\hline 340160.00 & $\mathrm{~K}=10$ & 340160.00 & 100.00 & 0.24 & 1.14 & 36 \\
\hline 338864.25 & $K=20$ & 340160.00 & 100.00 & 0.26 & 1.23 & 36 \\
\hline \multirow{2}{*}{0.30} & $\mathrm{~K}=30$ & 340160.00 & 100.00 & 0.29 & 1.27 & 36 \\
\hline & $\mathrm{K}=60$ & 340160.00 & 100.00 & 1.19 & 2.09 & 36 \\
\hline & $1: 50$-cuts $\star$ & 55590.96 & 9.23 & 94.33 & 880.95 & 427 \\
\hline air04 & GMI & 55583.78 & 8.04 & 2.02 & 335.94 & 202 \\
\hline 56137.00 & $\mathrm{~K}=10 \star$ & 55580.69 & 7.52 & 3.16 & 514.19 & 283 \\
\hline 55535.44 & $K=20 \star$ & 55583.86 & 8.05 & 5.93 & 510.79 & 300 \\
\hline \multirow[t]{2}{*}{34.82} & $\mathrm{~K}=30 \star$ & 55585.19 & 8.27 & 10.13 & 581.08 & 370 \\
\hline & $K=60 \star$ & 55586.21 & 8.44 & 197.07 & 723.21 & 389 \\
\hline & $1: 50$-cuts $\star$ & 25903.63 & 5.24 & 82.52 & 172.38 & 423 \\
\hline air05 & GMI & 25899.70 & 4.45 & 1.39 & 34.44 & 201 \\
\hline 26374.00 & $K=10 \star$ & 25898.87 & 4.28 & 2.62 & 44.05 & 229 \\
\hline 25877.61 & $\mathrm{~K}=20 \star$ & 25900.87 & 4.69 & 3.54 & 58.50 & 260 \\
\hline \multirow[t]{2}{*}{3.19} & $K=30 \star$ & 25900.96 & 4.70 & 9.10 & 95.19 & 298 \\
\hline & $\mathrm{K}=60 \star$ & 25902.02 & 4.92 & 177.51 & 307.77 & 386 \\
\hline & $1: 50$-cuts & -2451470.55 & 41.65 & 2.65 & 40.17 & 398 \\
\hline сар6000 & GMI & -2451470.55 & 41.65 & 0.05 & 0.59 & 9 \\
\hline-2451377.00 & $\mathrm{~K}=10$ & -2451474.58 & 39.14 & 0.08 & 0.73 & 11 \\
\hline-2451537.33 & $\mathrm{~K}=20$ & -2451474.58 & 39.14 & 0.12 & 0.78 & 11 \\
\hline \multirow[t]{2}{*}{0.33} & $\mathrm{~K}=30$ & -2451472.58 & 40.38 & 0.17 & 0.82 & 11 \\
\hline & $\mathrm{K}=60$ & -2451472.49 & 40.44 & 1.59 & 2.26 & 11 \\
\hline & $1: 50$-cuts $\star$ & 4665.16 & 13.40 & 1.62 & 3.13 & 200 \\
\hline 1152lav & GMI & 4664.41 & 12.25 & 0.05 & 0.40 & 51 \\
\hline 4722.00 & $\mathrm{~K}=10 \star$ & 4664.03 & 11.67 & 0.22 & 0.79 & 88 \\
\hline 4656.36 & $\mathrm{~K}=20 \star$ & 4664.60 & 12.54 & 0.52 & 1.17 & 88 \\
\hline \multirow[t]{2}{*}{0.09} & $\mathrm{~K}=30 \star$ & 4665.26 & 13.55 & 2.23 & 3.59 & 237 \\
\hline & $K=60 \star$ & 4665.87 & 14.48 & 83.55 & 86.58 & 349 \\
\hline & $1: 50$-cuts $\star$ & 996.97 & 56.88 & 0.07 & 0.09 & 300 \\
\hline lseu & GMI & 991.87 & 55.09 & 0.00 & 0.01 & 13 \\
\hline 1120.00 & $\mathrm{~K}=10 \star$ & 996.29 & 56.64 & 0.02 & 0.03 & 22 \\
\hline 834.68 & $K=20 \star$ & 997.34 & 57.01 & 0.07 & 0.08 & 25 \\
\hline \multirow{2}{*}{0.00} & $\mathrm{~K}=30 \star$ & 998.64 & 57.47 & 0.41 & 0.42 & 42 \\
\hline & $\mathrm{K}=60 \star$ & 1000.29 & 58.04 & 8.17 & 8.19 & 31 \\
\hline & $1: 50$-cuts & -13164.00 & 100.00 & 4798.42 & 4884.79 & 10275 \\
\hline manna81 & GMI & -13164.00 & 100.00 & 13.58 & 16.55 & 812 \\
\hline-13164.00 & $\mathrm{~K}=10$ & -13164.00 & 100.00 & 21.28 & 24.32 & 812 \\
\hline-13297.00 & $\mathrm{~K}=20$ & -13164.00 & 100.00 & 21.95 & 25.06 & 812 \\
\hline \multirow[t]{2}{*}{0.13} & $\mathrm{~K}=30$ & -13164.00 & 100.00 & 23.74 & 27.13 & 812 \\
\hline & $\mathrm{K}=60$ & -13164.00 & 100.00 & 33.91 & 38.17 & 812 \\
\hline & 1:50-cuts & 115081.21 & 82.20 & 4057.42 & 4244.84 & 14200 \\
\hline mitre & GMI & 115081.21 & 82.20 & 12.71 & 15.95 & 552 \\
\hline 115155.00 & $\mathrm{~K}=10$ & 115067.88 & 78.98 & 21.18 & 25.85 & 662 \\
\hline 114740.52 & $K=20$ & 115081.21 & 82.20 & 60.69 & 67.74 & 725 \\
\hline \multirow[t]{2}{*}{0.34} & $\mathrm{~K}=30$ & 115081.21 & 82.20 & 72.65 & 80.40 & 701 \\
\hline & $\mathrm{K}=60$ & 115081.21 & 82.20 & 573.16 & 583.40 & 738 \\
\hline & $1: 50$-cuts & 6535.50 & 21.47 & 1.54 & 3.49 & 200 \\
\hline $\bmod 010$ & GMI & 6535.50 & 21.47 & 0.05 & 0.42 & 34 \\
\hline 6548.00 & $\mathrm{~K}=10$ & 6535.46 & 21.24 & 0.14 & 0.67 & 38 \\
\hline 6532.08 & $\mathrm{~K}=20$ & 6535.46 & 21.24 & 0.17 & 0.66 & 36 \\
\hline \multirow[t]{2}{*}{0.09} & $\mathrm{~K}=30 \star$ & 6535.75 & 23.04 & 0.26 & 0.70 & 36 \\
\hline & $\mathrm{K}=60 \star$ & 6536.00 & 24.61 & 2.88 & 3.40 & 40 \\
\hline & $1: 50$-cuts $\star$ & 2843.74 & 56.85 & 0.00 & 0.02 & 219 \\
\hline p0033 & GMI & 2830.95 & 54.60 & 0.00 & 0.00 & 8 \\
\hline 3089.00 & $K=10 \star$ & 2629.39 & 19.14 & 0.02 & 0.03 & 15 \\
\hline 2520.57 & $K=20 \star$ & 2690.91 & 29.97 & 0.07 & 0.07 & 18 \\
\hline 0.00 & $\mathrm{~K}=30 \star$ & 2749.28 & 40.23 & 0.33 & 0.34 & 22 \\
\hline & $\mathrm{K}=60 \star$ & 2842.25 & 56.59 & 4.73 & 4.73 & 19 \\
\hline & $1: 50$-cuts $\star$ & 7007.91 & 17.96 & 0.14 & 0.22 & 200 \\
\hline p0201 & GMI & 7000.56 & 16.97 & 0.00 & 0.02 & 22 \\
\hline 7615.00 & $K=10 \star$ & 7002.42 & 17.22 & 0.03 & 0.08 & 61 \\
\hline 6875.00 & $K=20 \star$ & 7002.43 & 17.22 & 0.05 & 0.13 & 71 \\
\hline 0.01 & $\mathrm{~K}=30 \star$ & 7004.88 & 17.55 & 0.24 & 0.30 & 76 \\
\hline & $K=60 \star$ & 7004.96 & 17.56 & 2.54 & 2.61 & 86 \\
\hline & $1: 50$-cuts & 179882.58 & 3.70 & 0.45 & 0.53 & 400 \\
\hline p0282 & GMI & 179882.58 & 3.70 & 0.00 & 0.02 & 32 \\
\hline 258411.00 & $\mathrm{~K}=10$ & 179711.49 & 3.49 & 0.04 & 0.05 & 38 \\
\hline 176867.50 & $K=20$ & 179830.00 & 3.63 & 0.10 & 0.12 & 43 \\
\hline 0.00 & $\mathrm{~K}=30$ & 179784.53 & 3.58 & 0.41 & 0.44 & 45 \\
\hline & $K=60$ & 179830.00 & 3.63 & 5.90 & 5.93 & 43 \\
\hline
\end{tabular}


Table 6: MIPLIB pure integer problems (part 2).

\begin{tabular}{|c|c|c|c|c|c|c|}
\hline Problem & $\begin{array}{c}\text { Type of } \\
\text { Cuts }\end{array}$ & Final LB & $\begin{array}{c}\text { Closed } \\
\text { Gap } \\
(\%)\end{array}$ & $\begin{array}{c}\text { Separation } \\
\text { Time } \\
\text { (seconds) }\end{array}$ & $\begin{array}{c}\text { Total } \\
\text { Time } \\
\text { (seconds) }\end{array}$ & $\begin{array}{c}\text { Number of } \\
\text { Cuts }\end{array}$ \\
\hline & 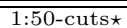 & 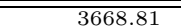 & 40.04 & 3.80 & 4.49 & 22113 \\
\hline p0548 & GMI & 3667.64 & 40.02 & 0.02 & 0.06 & 55 \\
\hline 8691.00 & $\mathrm{~K}=10 \star$ & 738.79 & 5.06 & 0.09 & 0.14 & 88 \\
\hline 315.25 & $\mathrm{~K}=20 \star$ & 3258.85 & 35.14 & 0.29 & 0.35 & 99 \\
\hline \multirow[t]{2}{*}{0.01} & $\mathrm{~K}=30 \star$ & 3139.64 & 33.72 & 1.09 & 1.16 & 102 \\
\hline & $\mathrm{K}=60 \star$ & 3493.37 & 37.94 & 23.29 & 23.40 & 113 \\
\hline & $1: 50$-cuts $\star$ & 2691.42 & 0.61 & 8.24 & 10.35 & 1300 \\
\hline p2756 & GMI & 2691.09 & 0.54 & 0.08 & 0.16 & 35 \\
\hline 3124.00 & $\mathrm{~K}=10 \star$ & 2689.80 & 0.24 & 0.24 & 0.44 & 57 \\
\hline 2688.75 & $\mathrm{~K}=20 \star$ & 2690.00 & 0.29 & 0.34 & 0.54 & 61 \\
\hline \multirow[t]{2}{*}{0.02} & $\mathrm{~K}=30 \star$ & 2690.00 & 0.29 & 0.56 & 0.76 & 62 \\
\hline & $\mathrm{K}=60 \star$ & 2690.00 & 0.29 & 8.65 & 8.81 & 65 \\
\hline & $1: 50$-cuts & 405.14 & 6.75 & 978.83 & 1019.77 & 676 \\
\hline seymour & GMI & 405.14 & 6.75 & 16.09 & 30.95 & 276 \\
\hline 423.00 & $\mathrm{~K}=10$ & 405.13 & 6.69 & 26.64 & 45.43 & 410 \\
\hline 403.85 & $\mathrm{~K}=20$ & 405.13 & 6.72 & 27.83 & 45.03 & 427 \\
\hline \multirow[t]{2}{*}{24.01} & $\mathrm{~K}=30$ & 405.14 & 6.75 & 51.41 & 69.78 & 401 \\
\hline & $\mathrm{K}=60$ & 405.14 & 6.75 & 290.60 & 305.18 & 348 \\
\hline & $1: 50$-cuts & -5000.00 & $(-)$ & 43.27 & 72.82 & 200 \\
\hline disctom & GMI & -5000.00 & $(-)$ & 1.45 & 27.86 & 200 \\
\hline-5000.00 & $\mathrm{~K}=10$ & -5000.00 & $(-)$ & 2.16 & 28.47 & 200 \\
\hline-5000.00 & $\mathrm{~K}=20$ & -5000.00 & $(-)$ & 2.48 & 28.97 & 200 \\
\hline \multirow[t]{2}{*}{1.25} & $\mathrm{~K}=30$ & -5000.00 & $(-)$ & 3.11 & 29.69 & 200 \\
\hline & $\mathrm{K}=60$ & -5000.00 & $(-)$ & 13.84 & 40.28 & 200 \\
\hline & $1: 50$-cuts & 0.00 & $(-)$ & 0.02 & 0.05 & 200 \\
\hline enigma & GMI & 0.00 & $(-)$ & 0.00 & 0.00 & 8 \\
\hline 0.00 & $\mathrm{~K}=10$ & 0.00 & $(-)$ & 0.00 & 0.00 & 8 \\
\hline 0.00 & $\mathrm{~K}=20$ & 0.00 & $(-)$ & 0.01 & 0.01 & 8 \\
\hline \multirow[t]{2}{*}{0.00} & $\mathrm{~K}=30$ & 0.00 & $(-)$ & 0.04 & 0.04 & 8 \\
\hline & $\mathrm{K}=60$ & 0.00 & $(-)$ & 0.62 & 0.63 & 8 \\
\hline & $1: 50$-cuts $\star$ & -74219846.41 & 29.43 & 5.45 & 27.53 & 653 \\
\hline harp2 & GMI & -74251958.32 & 22.35 & 0.04 & 0.19 & 30 \\
\hline-73899798.00 & $\mathrm{~K}=10 \star$ & -74247224.08 & 23.40 & 0.23 & 0.69 & 58 \\
\hline-74353341.50 & $\mathrm{~K}=20 \star$ & -74236993.08 & 25.65 & 0.35 & 0.97 & 62 \\
\hline \multirow[t]{2}{*}{0.04} & $\mathrm{~K}=30 \star$ & -74236058.30 & 25.86 & 0.93 & 1.71 & 71 \\
\hline & $\mathrm{K}=60 \star \star$ & -74225928.01 & 28.09 & 26.86 & 27.79 & 75 \\
\hline & $1: 50$-cuts & 13.00 & 0.00 & 0.38 & 0.40 & 209 \\
\hline stein27 & GMI & 13.00 & 0.00 & 0.01 & 0.01 & 84 \\
\hline 18.00 & $\mathrm{~K}=10$ & 13.00 & 0.00 & 0.03 & 0.04 & 84 \\
\hline 13.00 & $\mathrm{~K}=20$ & 13.00 & 0.00 & 0.07 & 0.07 & 84 \\
\hline \multirow[t]{2}{*}{0.00} & $\mathrm{~K}=30$ & 13.00 & 0.00 & 0.17 & 0.18 & 84 \\
\hline & $\mathrm{K}=60$ & 13.00 & 0.00 & 2.31 & 2.34 & 84 \\
\hline & $1: 50$-cuts & 22.00 & 0.00 & 1.26 & 1.29 & 200 \\
\hline stein 45 & GMI & 22.00 & 0.00 & 0.04 & 0.09 & 200 \\
\hline 30.00 & $\mathrm{~K}=10$ & 22.00 & 0.00 & 0.10 & 0.14 & 200 \\
\hline 22.00 & $\mathrm{~K}=20$ & 22.00 & 0.00 & 0.21 & 0.26 & 200 \\
\hline \multirow{2}{*}{0.01} & $\mathrm{~K}=30$ & 22.00 & 0.00 & 0.50 & 0.52 & 200 \\
\hline & $\mathrm{K}=60$ & 22.00 & 0.00 & 5.03 & 5.06 & 200 \\
\hline
\end{tabular}


Table 7: Atamtürk's bounded problems (averages over 5 instances).

\begin{tabular}{|c|c|c|c|c|c|}
\hline Set & $\begin{array}{c}\text { Type of } \\
\text { Cuts }\end{array}$ & $\begin{array}{c}\text { Closed } \\
\text { Gap } \\
(\%) \\
\end{array}$ & $\begin{array}{c}\text { Separation } \\
\text { Time } \\
\text { (seconds) }\end{array}$ & $\begin{array}{c}\text { Total } \\
\text { Time } \\
\text { (seconds) }\end{array}$ & $\begin{array}{l}\text { Average } \\
\text { Number } \\
\text { of Cuts }\end{array}$ \\
\hline \multirow{7}{*}{ mik.250-10-100 } & $1: 50$-cuts $\star$ & 74.69 & 3.501 & 8.294 & 2948.40 \\
\hline & GMI & 67.41 & 0.012 & 0.034 & 100.00 \\
\hline & $\mathrm{k}=5$ & 66.60 & 0.052 & 0.182 & 174.60 \\
\hline & $\mathrm{k}=10$ & 66.78 & 0.098 & 0.266 & 204.60 \\
\hline & $\mathrm{k}=20 \star$ & 67.52 & 0.405 & 0.623 & 227.40 \\
\hline & $\mathrm{k}=30 \star$ & 70.10 & 2.311 & 2.622 & 257.00 \\
\hline & $\mathrm{k}=60 \star$ & 72.02 & 64.913 & 65.314 & 289.20 \\
\hline \multirow{7}{*}{ mik.250-10-50 } & $1: 50$-cuts $\star$ & 76.34 & 1.975 & 23.976 & 2100.60 \\
\hline & GMI & 50.87 & 0.012 & 0.022 & 50.00 \\
\hline & $\mathrm{k}=5$ & 50.37 & 0.026 & 0.118 & 86.00 \\
\hline & $\mathrm{k}=10$ & 50.37 & 0.066 & 0.180 & 103.40 \\
\hline & $\mathrm{k}=20 \star$ & 51.14 & 0.216 & 0.363 & 114.00 \\
\hline & $\mathrm{k}=30 \star$ & 55.62 & 0.993 & 1.206 & 132.00 \\
\hline & $\mathrm{k}=60 \star$ & 66.04 & 35.559 & 35.898 & 163.40 \\
\hline \multirow{7}{*}{ mik.250-10-75 } & $1: 50$-cuts $\star$ & 72.54 & 3.179 & 14.369 & 2795.80 \\
\hline & GMI & 56.19 & 0.016 & 0.034 & 75.00 \\
\hline & $\mathrm{k}=5$ & 55.18 & 0.048 & 0.198 & 130.20 \\
\hline & $\mathrm{k}=10$ & 55.33 & 0.070 & 0.268 & 153.00 \\
\hline & $\mathrm{k}=20$ 太 & 56.20 & 0.318 & 0.593 & 174.40 \\
\hline & $\mathrm{k}=30 \star$ & 60.78 & 1.741 & 2.155 & 200.20 \\
\hline & $\mathrm{k}=60 \star$ & 66.22 & 56.732 & 57.366 & 232.60 \\
\hline \multirow{7}{*}{ mik.250-20-100 } & $1: 50$-cuts $\star$ & 75.27 & 3.145 & 7.713 & 2689.80 \\
\hline & GMI & 70.46 & 0.022 & 0.044 & 100.00 \\
\hline & $\mathrm{k}=5$ & 69.83 & 0.048 & 0.182 & 174.60 \\
\hline & $\mathrm{k}=10$ & 69.99 & 0.096 & 0.248 & 204.00 \\
\hline & $\mathrm{k}=20$ & 70.42 & 0.423 & 0.643 & 229.20 \\
\hline & $\mathrm{k}=30 \star$ & 72.25 & 2.087 & 2.379 & 257.00 \\
\hline & $\mathrm{k}=60 \star$ & 73.68 & 71.585 & 72.052 & 286.40 \\
\hline \multirow{7}{*}{ mik.250-20-50 } & $1: 50$-cuts $\star$ & 74.77 & 1.929 & 19.041 & 2049.60 \\
\hline & GMI & 51.60 & 0.012 & 0.028 & 50.00 \\
\hline & $\mathrm{k}=5$ & 51.09 & 0.034 & 0.118 & 86.00 \\
\hline & $\mathrm{k}=10$ & 51.08 & 0.062 & 0.184 & 103.20 \\
\hline & $\mathrm{k}=20 \star$ & 51.87 & 0.234 & 0.389 & 115.40 \\
\hline & $\mathrm{k}=30 \star$ & 56.41 & 1.066 & 1.320 & 132.20 \\
\hline & $\mathrm{k}=60 \star$ & 66.12 & 37.179 & 37.594 & 166.60 \\
\hline \multirow{7}{*}{ mik.250-20-75 } & $1: 50$-cuts $\star$ & 74.37 & 2.936 & 12.135 & 2586.60 \\
\hline & GMI & 61.29 & 0.018 & 0.040 & 75.00 \\
\hline & $\mathrm{k}=5$ & 60.21 & 0.046 & 0.206 & 130.20 \\
\hline & $\mathrm{k}=10$ & 60.38 & 0.078 & 0.286 & 152.40 \\
\hline & $\mathrm{k}=20 \star$ & 61.35 & 0.334 & 0.637 & 176.80 \\
\hline & $\mathrm{k}=30 \star$ & 66.33 & 1.658 & 2.063 & 201.80 \\
\hline & $\mathrm{k}=60 \star$ & 70.14 & 54.352 & 55.023 & 241.20 \\
\hline \multirow{7}{*}{ mik.250-5-100 } & $1: 50$-cuts $\star$ & 71.17 & 3.990 & 9.996 & 3196.00 \\
\hline & GMI & 61.29 & 0.020 & 0.038 & 100.00 \\
\hline & $\mathrm{k}=5$ & 60.43 & 0.054 & 0.154 & 174.60 \\
\hline & $\mathrm{k}=10$ & 60.58 & 0.098 & 0.258 & 204.80 \\
\hline & $\mathrm{k}=20 \star$ & 61.43 & 0.413 & 0.633 & 229.00 \\
\hline & $\mathrm{k}=30 \star$ & 64.61 & 2.351 & 2.688 & 265.40 \\
\hline & $\mathrm{k}=60 \star$ & 67.35 & 75.697 & 76.123 & 297.00 \\
\hline
\end{tabular}


Table 8: Atamtürk's unbounded problems (averages over 5 instances).

\begin{tabular}{|c|c|c|c|c|c|}
\hline Set & $\begin{array}{l}\text { Type of } \\
\text { Cuts }\end{array}$ & $\begin{array}{c}\text { Closed } \\
\text { Gap } \\
(\%)\end{array}$ & $\begin{array}{c}\text { Separation } \\
\text { Time } \\
\text { (seconds) }\end{array}$ & $\begin{array}{c}\text { Total } \\
\text { Time } \\
\text { (seconds) }\end{array}$ & $\begin{array}{l}\text { Average } \\
\text { Number } \\
\text { of Cuts }\end{array}$ \\
\hline \multirow{7}{*}{ mik.250-1-100 } & $1: 50$-cuts & 74.55 & 3.898 & 9.474 & 3187.20 \\
\hline & GMI & 63.26 & 0.014 & 0.032 & 100.00 \\
\hline & $\mathrm{k}=5$ & 62.63 & 0.058 & 0.182 & 174.60 \\
\hline & $\mathrm{k}=10$ & 62.78 & 0.100 & 0.232 & 203.80 \\
\hline & $\mathrm{k}=20 \star$ & 63.28 & 0.411 & 0.619 & 225.80 \\
\hline & $\mathrm{k}=30 \star$ & 66.27 & 1.891 & 2.129 & 262.20 \\
\hline & $\mathrm{k}=60 \star$ & 70.83 & 64.801 & 65.294 & 303.20 \\
\hline \multirow{7}{*}{ mik.250-1-50 } & $1: 50$-cuts $\star$ & 78.52 & 2.117 & 23.440 & 2185.40 \\
\hline & GMI & 50.07 & 0.008 & 0.024 & 50.00 \\
\hline & $\mathrm{k}=5$ & 49.58 & 0.036 & 0.118 & 86.00 \\
\hline & $\mathrm{k}=10$ & 49.58 & 0.062 & 0.184 & 103.40 \\
\hline & $\mathrm{k}=20 \star$ & 50.34 & 0.232 & 0.379 & 114.00 \\
\hline & $\mathrm{k}=30 \star$ & 54.74 & 0.993 & 1.234 & 132.60 \\
\hline & $\mathrm{k}=60 \star$ & 65.52 & 35.902 & 36.212 & 157.20 \\
\hline \multirow{7}{*}{ mik.250-1-75 } & $1: 50$-cuts $\star$ & 76.14 & 3.419 & 19.087 & 3014.40 \\
\hline & GMI & 54.83 & 0.018 & 0.034 & 75.00 \\
\hline & $\mathrm{k}=5$ & 53.82 & 0.046 & 0.176 & 130.20 \\
\hline & $\mathrm{k}=10$ & 53.97 & 0.086 & 0.282 & 153.00 \\
\hline & $\mathrm{k}=20$ & 54.82 & 0.312 & 0.589 & 174.40 \\
\hline & $\mathrm{k}=30 \star$ & 59.53 & 1.642 & 2.043 & 199.00 \\
\hline & $\mathrm{k}=60 \star$ & 67.45 & 56.796 & 57.483 & 236.00 \\
\hline \multirow{7}{*}{ mik.500-1-100 } & $1: 50$-cuts $\star$ & 75.13 & 10.467 & 83.001 & 3293.20 \\
\hline & GMI & 63.48 & 0.046 & 0.130 & 100.00 \\
\hline & $\mathrm{k}=5$ & 62.42 & 0.124 & 2.175 & 174.60 \\
\hline & $\mathrm{k}=10$ & 62.53 & 0.204 & 2.636 & 206.40 \\
\hline & $\mathrm{k}=20 \star$ & 63.57 & 0.571 & 3.112 & 224.80 \\
\hline & $\mathrm{k}=30 \star$ & 68.01 & 2.221 & 5.772 & 267.40 \\
\hline & $\mathrm{k}=60 \star$ & 71.92 & 76.314 & 84.912 & 321.40 \\
\hline \multirow{7}{*}{ mik.500-1-50 } & $1: 50$-cuts $\star$ & 76.67 & 4.418 & 75.238 & 2117.20 \\
\hline & GMI & 50.68 & 0.024 & 0.080 & 50.00 \\
\hline & $\mathrm{k}=5$ & 50.19 & 0.062 & 0.401 & 86.00 \\
\hline & $\mathrm{k}=10$ & 50.19 & 0.102 & 0.479 & 102.80 \\
\hline & $\mathrm{k}=20 \star$ & 50.88 & 0.284 & 0.793 & 113.60 \\
\hline & $\mathrm{k}=30 \star$ & 55.41 & 1.096 & 1.961 & 133.60 \\
\hline & $\mathrm{k}=60 \star$ & 66.10 & 37.354 & 38.610 & 157.80 \\
\hline \multirow{7}{*}{ mik.500-1-75 } & $1: 50$-cuts $\star$ & 74.92 & 8.360 & 91.904 & 2932.40 \\
\hline & GMI & 55.13 & 0.034 & 0.102 & 75.00 \\
\hline & $\mathrm{k}=5$ & 54.12 & 0.098 & 1.248 & 130.20 \\
\hline & $\mathrm{k}=10$ & 54.28 & 0.160 & 1.364 & 151.40 \\
\hline & $\mathrm{k}=20$ & 55.08 & 0.431 & 2.135 & 174.80 \\
\hline & $\mathrm{k}=30 \star$ & 59.91 & 1.600 & 4.094 & 197.20 \\
\hline & $\mathrm{k}=60 \star$ & 67.48 & 50.757 & 54.979 & 240.40 \\
\hline
\end{tabular}


Table 9: Random knapsack problems with coefficients in $\{1, \ldots, 1000\}$.

\begin{tabular}{|c|c|c|c|c|c|c|c|c|c|}
\hline \multirow[b]{2}{*}{$n$} & \multirow{2}{*}{$\begin{array}{l}\text { Type } \\
\text { of } \\
\text { Cuts }\end{array}$} & \multicolumn{4}{|c|}{ Binary } & \multicolumn{4}{|c|}{ Bounded } \\
\hline & & $\begin{array}{c}\text { Closed } \\
\text { Gap } \\
(\%)\end{array}$ & $\begin{array}{l}\text { Sep. } \\
\text { Time } \\
\text { (sec) }\end{array}$ & $\begin{array}{l}\text { Tot. } \\
\text { Time } \\
\text { (sec) }\end{array}$ & $\begin{array}{l}\text { Avg. } \\
\text { Num. } \\
\text { of } \\
\text { Cuts }\end{array}$ & $\begin{array}{c}\text { Closed } \\
\text { Gap } \\
(\%)\end{array}$ & $\begin{array}{l}\text { Sep. } \\
\text { Time } \\
(\mathrm{sec})\end{array}$ & $\begin{array}{l}\text { Tot. } \\
\text { Time } \\
\text { (sec) }\end{array}$ & $\begin{array}{l}\text { Avg. } \\
\text { Num. } \\
\text { of } \\
\text { Cuts }\end{array}$ \\
\hline \multirow{7}{*}{10} & $1: 50$-cuts & $\overline{90.46 \star}$ & 0.000 & 0.002 & 49.93 & $94.75 \star$ & 0.000 & 0.000 & 50.00 \\
\hline & GMI & 76.15 & 0.000 & 0.000 & 1.00 & 74.47 & 0.000 & 0.001 & 1.00 \\
\hline & $\mathrm{k}=5$ & 65.92 & 0.001 & 0.001 & 1.97 & 71.49 & 0.000 & 0.001 & 2.00 \\
\hline & $\mathrm{k}=10$ & $81.27 \star$ & 0.001 & 0.002 & 2.83 & $82.10 \star$ & 0.001 & 0.003 & 2.63 \\
\hline & $\mathrm{k}=20$ & $89.30 \star$ & 0.003 & 0.006 & 3.33 & $91.25 \star$ & 0.004 & 0.007 & 3.23 \\
\hline & $\mathrm{k}=30$ & $91.51 \star$ & 0.014 & 0.016 & 3.57 & $94.08 \star$ & 0.012 & 0.016 & 3.80 \\
\hline & $\mathrm{k}=60$ & $92.90 \star$ & 0.229 & 0.232 & 3.50 & $97.36 \star$ & 0.249 & 0.252 & 4.13 \\
\hline \multirow{7}{*}{50} & $1: 50$-cuts & $79.94 \star$ & 0.002 & 0.007 & 49.90 & $88.83 \star$ & 0.001 & 0.003 & 49.93 \\
\hline & GMI & 50.71 & 0.000 & 0.001 & 1.00 & 42.44 & 0.000 & 0.001 & 1.00 \\
\hline & $\mathrm{k}=5$ & $52.00 \star$ & 0.001 & 0.002 & 2.07 & 36.46 & 0.001 & 0.002 & 1.73 \\
\hline & $\mathrm{k}=10$ & $62.63 \star$ & 0.001 & 0.005 & 3.20 & $46.62 \star$ & 0.002 & 0.003 & 2.67 \\
\hline & $\mathrm{k}=20$ & $69.44 \star$ & 0.005 & 0.008 & 3.90 & $68.21 \star$ & 0.005 & 0.009 & 3.77 \\
\hline & $\mathrm{k}=30$ & $70.34 \star$ & 0.016 & 0.020 & 4.97 & $73.74 \star$ & 0.020 & 0.023 & 4.63 \\
\hline & $\mathrm{k}=60$ & $77.78 \star$ & 0.294 & 0.300 & 5.63 & $83.88 \star$ & 0.360 & 0.365 & 5.67 \\
\hline \multirow{7}{*}{100} & $1: 50$-cuts & $78.41 \star$ & 0.002 & 0.009 & 49.97 & $82.84 \star$ & 0.002 & 0.008 & 49.43 \\
\hline & GMI & 39.80 & 0.000 & 0.000 & 1.00 & 39.31 & 0.000 & 0.001 & 1.00 \\
\hline & $\mathrm{k}=5$ & 34.88 & 0.001 & 0.003 & 1.77 & 34.12 & 0.002 & 0.004 & 1.77 \\
\hline & $\mathrm{k}=10$ & $49.98 \star$ & 0.001 & 0.003 & 2.80 & $44.71 \star$ & 0.001 & 0.002 & 2.60 \\
\hline & $\mathrm{k}=20$ & $58.65 \star$ & 0.007 & 0.011 & 4.27 & $59.39 \star$ & 0.007 & 0.008 & 4.20 \\
\hline & $\mathrm{k}=30$ & $66.57 \star$ & 0.019 & 0.024 & 5.50 & $67.65 \star$ & 0.016 & 0.022 & 4.80 \\
\hline & $\mathrm{k}=60$ & $74.88 \star$ & 0.347 & 0.351 & 6.73 & $75.64 \star$ & 0.357 & 0.363 & 6.23 \\
\hline \multirow{7}{*}{500} & $1: 50$-cuts & $74.09 \star$ & 0.014 & 0.062 & 49.60 & $75.99 \star$ & 0.012 & 0.060 & 49.93 \\
\hline & GMI & 26.20 & 0.000 & 0.003 & 1.00 & 22.44 & 0.000 & 0.004 & 1.00 \\
\hline & $\mathrm{k}=5$ & $26.69 \star$ & 0.003 & 0.007 & 1.93 & 19.90 & 0.001 & 0.006 & 1.80 \\
\hline & $\mathrm{k}=10$ & $34.13 \star$ & 0.005 & 0.009 & 3.27 & $25.08 \star$ & 0.002 & 0.008 & 2.77 \\
\hline & $\mathrm{k}=20$ & $43.08 \star$ & 0.010 & 0.017 & 4.87 & $37.03 \star$ & 0.008 & 0.015 & 3.90 \\
\hline & $\mathrm{k}=30$ & $48.74 \star$ & 0.024 & 0.032 & 5.57 & $41.72 \star$ & 0.019 & 0.027 & 4.50 \\
\hline & $\mathrm{k}=60$ & $60.09 \star$ & 0.366 & 0.384 & 7.33 & $54.93 \star$ & 0.329 & 0.341 & 6.20 \\
\hline \multirow{7}{*}{1000} & $1: 50$-cuts & $56.97 \star$ & 0.027 & 0.153 & 50.00 & $70.16 \star$ & 0.030 & 0.152 & 49.13 \\
\hline & GMI & 24.68 & 0.000 & 0.008 & 1.00 & 23.97 & 0.001 & 0.008 & 1.00 \\
\hline & $\mathrm{k}=5$ & 22.09 & 0.003 & 0.012 & 2.17 & 22.51 & 0.003 & 0.012 & 2.10 \\
\hline & $\mathrm{k}=10$ & $29.17 \star$ & 0.004 & 0.018 & 3.23 & $27.08 \star$ & 0.004 & 0.015 & 2.90 \\
\hline & $\mathrm{k}=20$ & $36.07 \star$ & 0.011 & 0.026 & 4.70 & $35.10 \star$ & 0.007 & 0.020 & 4.23 \\
\hline & $\mathrm{k}=30$ & $40.03 \star$ & 0.027 & 0.048 & 5.67 & $39.40 \star$ & 0.031 & 0.045 & 5.30 \\
\hline & $\mathrm{k}=60$ & $47.21 \star$ & 0.375 & 0.395 & 7.47 & $44.74 \star$ & 0.324 & 0.344 & 5.90 \\
\hline \multirow{7}{*}{5000} & $1: 50$-cuts & $47.07 \star$ & 0.156 & 0.910 & 49.90 & $53.96 \star$ & 0.156 & 0.904 & 49.97 \\
\hline & GMI & 17.03 & 0.004 & 0.063 & 1.00 & 12.78 & 0.002 & 0.060 & 1.00 \\
\hline & $\mathrm{k}=5$ & 15.69 & 0.008 & 0.082 & 2.03 & 10.74 & 0.008 & 0.081 & 2.00 \\
\hline & $\mathrm{k}=10$ & $19.50 \star$ & 0.013 & 0.102 & 3.07 & $19.49 \star$ & 0.012 & 0.099 & 3.10 \\
\hline & $\mathrm{k}=20$ & $25.86 \star$ & 0.021 & 0.140 & 4.63 & $25.01 \star$ & 0.022 & 0.127 & 4.20 \\
\hline & $\mathrm{k}=30$ & $29.64 \star$ & 0.036 & 0.179 & 5.53 & $28.31 \star$ & 0.034 & 0.159 & 4.93 \\
\hline & $\mathrm{k}=60$ & $36.53 \star$ & 0.433 & 0.656 & 8.43 & $36.29 \star$ & 0.381 & 0.543 & 6.43 \\
\hline \multirow{7}{*}{10000} & $1: 50$-cuts & $38.59 \star$ & 0.313 & 2.249 & 49.97 & $48.81 \star$ & 0.306 & 2.214 & 49.93 \\
\hline & GMI & 10.82 & 0.006 & 0.246 & 1.00 & 18.34 & 0.004 & 0.235 & 1.00 \\
\hline & $\mathrm{k}=5$ & 10.43 & 0.016 & 0.286 & 1.93 & 16.05 & 0.012 & 0.261 & 1.77 \\
\hline & $\mathrm{k}=10$ & $15.40 \star$ & 0.023 & 0.331 & 2.97 & $20.37 \star$ & 0.019 & 0.304 & 2.83 \\
\hline & $\mathrm{k}=20$ & $20.40 \star$ & 0.031 & 0.380 & 4.03 & $28.24 \star$ & 0.031 & 0.345 & 3.97 \\
\hline & $\mathrm{k}=30$ & $23.55 \star$ & 0.055 & 0.483 & 5.60 & $30.90 \star$ & 0.050 & 0.418 & 4.87 \\
\hline & $\mathrm{k}=60$ & $28.14 \star$ & 0.425 & 0.927 & 6.77 & $37.03 \star$ & 0.359 & 0.852 & 6.70 \\
\hline
\end{tabular}


Table 10: Random knapsack problems with coefficients in $\{1, \ldots, 100\}$.

\begin{tabular}{|c|c|c|c|c|c|c|c|c|c|}
\hline \multirow[b]{2}{*}{$n$} & \multirow{2}{*}{$\begin{array}{l}\text { Type } \\
\text { of } \\
\text { Cuts }\end{array}$} & \multicolumn{4}{|c|}{ Binary } & \multicolumn{4}{|c|}{ Bounded } \\
\hline & & $\begin{array}{c}\text { Closed } \\
\text { Gap } \\
(\%)\end{array}$ & $\begin{array}{l}\text { Sep. } \\
\text { Time } \\
(\text { sec) }\end{array}$ & $\begin{array}{l}\text { Tot. } \\
\text { Time } \\
(\text { sec) }\end{array}$ & $\begin{array}{c}\text { Avg. } \\
\text { Num. } \\
\text { of } \\
\text { Cuts }\end{array}$ & $\begin{array}{c}\text { Closed } \\
\text { Gap } \\
(\%)\end{array}$ & $\begin{array}{l}\text { Sep. } \\
\text { Time } \\
\text { (sec) }\end{array}$ & $\begin{array}{l}\text { Tot. } \\
\text { Time } \\
\text { (sec) }\end{array}$ & $\begin{array}{l}\text { Avg. } \\
\text { Num. } \\
\text { of } \\
\text { Cuts }\end{array}$ \\
\hline \multirow{8}{*}{10} & $1: 50$-cuts & 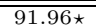 & 0.001 & 0.002 & 47.03 & $\overline{~ 96.54 \star}$ & 0.000 & 0.002 & 48.37 \\
\hline & GMI & 77.61 & 0.000 & 0.002 & 1.00 & 74.98 & 0.000 & 0.000 & 1.00 \\
\hline & $\mathrm{k}=5$ & 71.12 & 0.001 & 0.002 & 1.93 & 74.15 & 0.001 & 0.003 & 1.90 \\
\hline & $\mathrm{k}=10$ & $84.14 \star$ & 0.001 & 0.005 & 2.73 & $85.52 \star$ & 0.002 & 0.004 & 2.70 \\
\hline & $\mathrm{k}=20$ & $92.12 \star$ & 0.003 & 0.006 & 3.23 & $92.18 \star$ & 0.005 & 0.008 & 3.37 \\
\hline & $\mathrm{k}=30$ & $93.30 \star$ & 0.011 & 0.014 & 3.33 & $94.11 \star$ & 0.014 & 0.017 & 3.83 \\
\hline & $\mathrm{k}=60$ & $95.91 \star$ & 0.216 & 0.221 & 3.70 & $96.37 \star$ & 0.255 & 0.260 & 3.87 \\
\hline & Ideal k & $96.92 \star$ & 1.304 & 1.310 & 2.97 & $98.12 \star$ & 1.251 & 1.256 & 3.20 \\
\hline \multirow{8}{*}{50} & $1: 50$-cuts & $85.56 \star$ & 0.002 & 0.005 & 49.60 & $991.38 \star$ & 0.001 & 0.006 & 49.13 \\
\hline & GMI & 53.89 & 0.000 & 0.001 & 1.00 & 43.37 & 0.000 & 0.001 & 1.00 \\
\hline & $\mathrm{k}=5$ & $55.43 \star$ & 0.000 & 0.001 & 2.10 & 37.62 & 0.000 & 0.001 & 1.73 \\
\hline & $\mathrm{k}=10$ & $65.72 \star$ & 0.002 & 0.005 & 2.80 & $49.61 \star$ & 0.003 & 0.004 & 2.30 \\
\hline & $\mathrm{k}=20$ & $73.96 \star$ & 0.007 & 0.009 & 3.80 & $67.45 \star$ & 0.006 & 0.011 & 4.00 \\
\hline & $\mathrm{k}=30$ & $78.80 \star$ & 0.014 & 0.022 & 4.70 & $73.92 \star$ & 0.017 & 0.022 & 4.50 \\
\hline & $\mathrm{k}=60$ & $85.47 \star$ & 0.330 & 0.337 & 5.33 & $84.98 \star$ & 0.327 & 0.331 & 5.17 \\
\hline & Ideal k & $90.29 \star$ & 1.235 & 1.239 & 4.07 & $97.80 \star$ & 1.399 & 1.406 & 4.13 \\
\hline \multirow{8}{*}{100} & $1: 50$-cuts & $82.53 \star$ & 0.003 & 0.008 & 48.48 & $89.38 \star$ & 0.003 & 0.009 & 48.72 \\
\hline & GMI & 39.52 & 0.000 & 0.002 & 1.00 & 43.97 & 0.000 & 0.002 & 1.00 \\
\hline & $\mathrm{k}=5$ & 36.32 & 0.002 & 0.004 & 1.86 & 41.22 & 0.000 & 0.002 & 1.83 \\
\hline & $\mathrm{k}=10$ & $50.66 \star$ & 0.002 & 0.005 & 2.90 & $50.27 \star$ & 0.002 & 0.005 & 2.76 \\
\hline & $\mathrm{k}=20$ & $64.36 \star$ & 0.005 & 0.011 & 4.83 & $67.06 \star$ & 0.007 & 0.011 & 3.79 \\
\hline & $\mathrm{k}=30$ & $71.79 \star$ & 0.020 & 0.027 & 5.83 & $77.06 \star$ & 0.020 & 0.027 & 4.59 \\
\hline & $\mathrm{k}=60$ & $80.30 \star$ & 0.387 & 0.398 & 6.55 & $85.03 \star$ & 0.353 & 0.357 & 5.76 \\
\hline & Ideal k & $92.37 \star$ & 2.042 & 2.050 & 5.17 & $93.85 \star$ & 1.615 & 1.624 & 4.93 \\
\hline \multirow{8}{*}{500} & $1: 50$-cuts & $67.69 \star$ & 0.016 & 0.057 & 48.97 & $87.92 \star$ & 0.012 & 0.055 & 49.30 \\
\hline & GMI & 21.93 & 0.002 & 0.006 & 1.00 & 25.72 & 0.000 & 0.004 & 1.00 \\
\hline & $\mathrm{k}=5$ & 20.79 & 0.003 & 0.008 & 1.90 & $26.68 \star$ & 0.001 & 0.006 & 1.80 \\
\hline & $\mathrm{k}=10$ & $28.33 \star$ & 0.002 & 0.010 & 3.03 & $36.09 \star$ & 0.002 & 0.009 & 3.00 \\
\hline & $\mathrm{k}=20$ & $40.85 \star$ & 0.009 & 0.020 & 4.45 & $49.99 \star$ & 0.009 & 0.019 & 4.57 \\
\hline & $\mathrm{k}=30$ & $46.50 \star$ & 0.024 & 0.034 & 5.03 & $53.87 \star$ & 0.023 & 0.036 & 5.17 \\
\hline & $\mathrm{k}=60$ & $56.12 \star$ & 0.357 & 0.372 & 6.45 & $69.85 \star$ & 0.415 & 0.430 & 6.83 \\
\hline & Ideal k & $73.68 \star$ & 1.756 & 1.768 & 6.34 & $96.80 \star$ & 2.636 & 2.652 & 7.13 \\
\hline \multirow{8}{*}{1000} & $1: 50$-cuts & $79.88 \star$ & 0.030 & 0.130 & 48.73 & $93.05 \star$ & 0.023 & 0.124 & 48.23 \\
\hline & GMI & 24.16 & 0.000 & 0.008 & 1.00 & 26.48 & 0.001 & 0.008 & 1.00 \\
\hline & $\mathrm{k}=5$ & 22.99 & 0.002 & 0.012 & 1.80 & 25.23 & 0.005 & 0.015 & 1.67 \\
\hline & $\mathrm{k}=10$ & $38.57 \star$ & 0.006 & 0.021 & 3.47 & $31.93 \star$ & 0.003 & 0.017 & 2.33 \\
\hline & $\mathrm{k}=20$ & $48.07 \star$ & 0.010 & 0.032 & 5.03 & $41.26 \star$ & 0.009 & 0.022 & 3.43 \\
\hline & $\mathrm{k}=30$ & $55.72 \star$ & 0.030 & 0.055 & 6.83 & $54.78 \star$ & 0.021 & 0.036 & 4.17 \\
\hline & $\mathrm{k}=60$ & $65.81 \star$ & 0.363 & 0.390 & 8.03 & $73.97 \star$ & 0.377 & 0.402 & 5.97 \\
\hline & Ideal k & $89.54 \star$ & 2.036 & 2.067 & 6.63 & $100.00 \star$ & 1.727 & 1.749 & 5.40 \\
\hline \multirow{8}{*}{5000} & $1: 50$-cuts & $100.00 \star$ & 0.149 & 0.823 & 47.93 & $100.00 \star$ & 0.125 & 0.814 & 47.70 \\
\hline & GMI & 19.67 & 0.004 & 0.068 & 1.00 & 10.38 & 0.004 & 0.067 & 1.00 \\
\hline & $\mathrm{k}=5$ & $20.91 \star$ & 0.010 & 0.088 & 1.77 & $11.10 \star$ & 0.009 & 0.083 & 1.47 \\
\hline & $\mathrm{k}=10$ & $28.81 \star$ & 0.018 & 0.105 & 2.60 & $19.98 \star$ & 0.015 & 0.101 & 2.40 \\
\hline & $\mathrm{k}=20$ & $39.70 \star$ & 0.026 & 0.126 & 3.47 & $25.30 \star$ & 0.025 & 0.130 & 3.57 \\
\hline & $\mathrm{k}=30$ & $42.99 \star$ & 0.042 & 0.163 & 4.50 & $33.11 \star$ & 0.039 & 0.165 & 4.53 \\
\hline & $\mathrm{k}=60$ & $55.66 \star$ & 0.340 & 0.485 & 5.40 & $47.19 \star$ & 0.429 & 0.637 & 7.27 \\
\hline & Ideal k & $100.00 \star$ & 1.909 & 2.036 & 4.33 & $100.00 \star$ & 1.834 & 1.968 & 4.93 \\
\hline \multirow{8}{*}{10000} & $1: 50$-cuts & $100.00 \star$ & 0.322 & 2.052 & 49.13 & $100.00 \star$ & 0.269 & 2.073 & 49.63 \\
\hline & GMI & 21.61 & 0.009 & 0.262 & 1.00 & 19.65 & 0.012 & 0.263 & 1.00 \\
\hline & $\mathrm{k}=5$ & 19.61 & 0.022 & 0.299 & 1.73 & $20.79 \star$ & 0.019 & 0.284 & 1.57 \\
\hline & $\mathrm{k}=10$ & $26.16 \star$ & 0.032 & 0.336 & 2.70 & $32.15 \star$ & 0.025 & 0.302 & 2.27 \\
\hline & $\mathrm{k}=20$ & $30.73 \star$ & 0.041 & 0.368 & 3.27 & $41.04 \star$ & 0.048 & 0.373 & 3.63 \\
\hline & $\mathrm{k}=30$ & $37.32 \star$ & 0.060 & 0.416 & 4.00 & $45.52 \star$ & 0.062 & 0.431 & 4.27 \\
\hline & $\mathrm{k}=60$ & $48.89 \star$ & 0.403 & 0.876 & 6.10 & $52.99 \star$ & 0.381 & 0.804 & 5.10 \\
\hline & Ideal $\mathrm{k}$ & $100.00 \star$ & 2.134 & 2.505 & 4.73 & $100.00 \star$ & 2.816 & 3.192 & 4.67 \\
\hline
\end{tabular}

